\title{
A Robust Model for the Ramp-Constrained Economic Dispatch Problem with Uncertain Renewable Energy
}

\author{
M. Mohsen Moarefdoost ${ }^{\mathrm{a}}$, Alberto J. Lamadrid ${ }^{\mathrm{b}, *}$, Luis F. Zuluaga ${ }^{\mathrm{a}}$ \\ ${ }^{a}$ Lehigh University, Department of Industrial and Systems Engineering, 200 West Packer Avenue, Bethlehem, PA \\ 18015, Bethlehem, PA, 18015, United States \\ ${ }^{b}$ Lehigh University, Department of Economics, Rauch Business Center, 621 Taylor Street, Bethlehem, PA, 18015, \\ United States
}

\begin{abstract}
The inherent uncertainty of renewable energy sources (RES) makes the solution to the electricity network's associated economical dispatch (ED) problem with network constraints challenging. In particular, the uncertainty in the power output of RES requires conventional generation units to ramp up and down more frequently to maintain the power balance and the reliability of the system. Typically, the RES power output uncertainty is modeled in ED problems by considering its potential future scenarios. However, this leads to an optimization problem that is difficult to solve for real-sized networks. Here, we propose an alternative way of considering the uncertainty of RES and the consequent ramping of conventional generation via a robust reformulation of the problem. In particular, we show that in typical real-world instances of the ED problem, the associated deterministic formulation of the robust problem can be solved efficiently for larger scale constrained electricity networks even when the underlying uncertainty distribution is not normal. Moreover, we show that our approach results on dispatch solutions that require less ramping than scenario-based solutions, with little trade-off on the long-term expected costs of the network dispatch. These results also provide insights about how RES penetration affects cost and dispatch policies in the electricity network. To illustrate our results, we present relevant numerical experiments on IEEE test networks.
\end{abstract}

Keywords: Chance Constrained Optimization, Electricity Network, Ramping Costs

\section{Introduction}

The increased penetration of uncertain and variable renewable generation adds a new dimension to the problem of balancing power (matching power supply and power demand), and requires more flexibility from controllable and dispatchable power generating units. In particular, these units must ramp up and down (referred as ramping), or stop and start (referred as cycling), more frequently to maintain the system in balance and provide reliable power. This ramping and cycling result in additional emissions from fossil-fuel power plants and wear-and-tear costs that range from $\$ 150$ million to $\$ 450$ million annually at $30 \%$ renewable penetration (Lefton et al., 2008; Kumar et al., 2012). As a result, these costs can reduce the value of the renewable energy by up to $2.4 \%$ (Jordan and Venkataraman, 2012). Therefore, effective mathematical models for

\footnotetext{
${ }^{*}$ Corresponding Author
} 
the economic dispatch (ED) problem are required to reduce these costs and increase the economic value of renewable resources. As the penetration of RES in electricity grids increases, this issue becomes ever more important.

The economic dispatch problem has been the focus of thorough research activity. Starting with the seminal formulation of Carpentier (1962), this subject has continuously incorporated advances in optimization techniques (Huneault and Galiana, 1991), mainly driven by its overall economic impact, estimated to range between 1 and 5 billion US dollars per year in the US and up to 20 billion US dollars per year globally (see Cain et al. (2012b) and the references therein).

In this work, we examine the effect of ramping costs and constraints on generation and dispatch policies from an optimization point of view. Specifically, we develop a chance constrained optimization model (cf., Luedtke et al., 2007) to analyze the effect of ramping in a network with uncertain renewable energy generators.

Typically, the RES power output uncertainty is modeled in ED problems by considering potential future scenarios of the RES output (e.g., Meibom et al., 2010; Papavasiliou et al., 2011; Troy et al., 2012; Lamadrid et al., 2014), using stochastic programming techniques (Birge and Louveaux, 1997). However, this leads to optimization problems that are difficult to solve for real-sized networks.

Here, we propose an alternative way of considering the uncertainty of RES and the consequent ramping of conventional generation via a robust reformulation of the problem. In particular, we show that in typical real-world instances of the ED problem, the associated deterministic formulation of the robust problem can be solved efficiently for larger scale constrained electricity networks even when the underlying uncertainty distribution is not normal. Unlike previously related work in which normality assumptions apply to the main source of uncertainty (e.g., demand, Bienstock et al. (2012)), in this problem we are interested in modeling RES output (e.g., wind, wave, solar), whose uncertainty cannot be realistically modeled as normal. We also focus on the management of the bulk electricity power, where the magnitude of demand uncertainties is much smaller than the one derived from RES production.

Using relevant numerical experiments on IEEE test networks, we show that our approach results on dispatch solutions that require less ramping than scenario-based solutions, with little trade-off on the long-term expected costs of the network dispatch. Furthermore, for larger networks, a feasible solution cannot be obtained via the sampling approach in a reasonable time. Instead, with the robust reformulation one can obtain solutions with the characteristics above.

The structure of this paper is as follows. In Section 2, we study the effect of ramping costs on the dispatch policies of renewable generators. In Sections 3 and 4, we develop mathematical optimization models to address the effect of ramping in power networks when there is uncertain power output from RES, particularly wind power plants. In Section 5, we present relevant numerical experiments on IEEE test networks. Finally, in Section 6, we provide concluding remarks.

\section{Ramping Costs and Renewable Dispatch Policies}

The majority of studies in the literature regarding ramping in power grids can be classified into two main streams. The first stream of the literature focuses on the wear and tear costs of ramping for fossil-fuel power plants (Shibli et al., 2001; Lefton et al., 2008; Lefton, 2011; Lefton and Hilleman, 2011; Lew et al., 2012, 2013). These works quantify and estimate the cost of ramping on different types of conventional power plants. Kumar et al. (2012) estimate ramping costs based 
on generation type and size by applying statistical and engineering accounting methods. In a similar study, Jordan and Venkataraman (2012) evaluate the increased cost of ramping due to the integration of wind and solar resources in the Western grid. Navid and Rosenwald (2012) provide an optimization model for managing ramping with high penetration of renewable resources.

In the second literature stream, called dynamic economic dispatch, researchers and practitioners study the effect of ramping costs and constraints on the optimal power dispatch. Here, the focus has been on optimally dispatching electricity while considering costs and constraints on the generators' ramp rate (Wang and Shahidehpour, 1995; Majumdar et al., 1996; Irisarri et al., 1998; Schuerger et al., 2013). Kumano (2011) studies a dynamic economic load dispatch problem with constraints related to unit output and its ramp rate. Sherestha et al. (2004) study the strategic use of ramping rates in power systems with price and demand volatility. They develop a set of ramping processes for ramping costs and rates for an economic dispatch problem, and investigate the impact of the ramping process. Tanaka (2006) proposes a quadratic cost function for ramping and develops an optimal control model to obtain the optimal pricing policy in the case of a steep change in electricity load. Similarly, Attaviriyanupap et al. (2002) propose a heuristic algorithm based on Sequential Quadratic Programming (SQP) for solving a dynamic economic dispatch problem with non-smooth ramping cost function. These works consider ramping costs and constraints in deterministic settings for dynamic economic dispatch or the dynamic optimal power flow problems. However, uncertainty is an important part of any realistic system. In the current study, we solve a stochastic dynamic economic dispatch problem with power flow and ramping costs and constraints. We consider penetration of volatile and uncertain renewable resources and propose an optimization framework to manage the power system under high penetration of wind power.

Frequent ramping up and/or ramping down of fossil-fueled power plants beyond the elastic range causes thermal and pressure stresses which are the main reasons for thermal creep, fatigue and creep-fatigue interaction damages (Lefton et al., 2008; Shibli et al., 2001; Lefton and Hilleman, 2011). Creep is a time dependent and permanent deformation of materials when they are subjected to stress at high temperatures; and fatigue is a failure occurring under cyclic stress. When a generator's component faces cyclic stresses under high temperature, this component also experiences creep-fatigue interaction damages, which amplifies the damaging effect of creep and fatigue (Viswanathan, 1989). These types of damages could result in reduced components' life and thus increased capital and maintenance costs. Additionally, ramping up and/or ramping down increases fuel inefficiency and thus fuel consumption and cost increase. Faster ramp rates mean increased costs, and the relationship is not linear (Kumar et al., 2012). Therefore, a realistic ramping cost function is nonlinear in the ramp rate. Specifically, we model this ramping cost as a quadratic function. This is in line with the previous literature (e.g., Tanaka (2006); Lamadrid et al. (2014)). Moreover, this assumption ensures that the formulated models can be solved to optimality with current solvers, thanks to the convexity of the ramping costs. Lastly, our approach hinges on the potential of current optimization solvers to find a solution for problems with convex quadratic or convex piece-wise linear objectives.

Ramping costs have a significant effect on the policies for dispatch of RES due to the fact that renewable generators have variable and uncertain power output at different timescales (Ela and O'Malley, 2012). This inherent variability and uncertainty requires frequent ramping on the conventional generators, and the incurred cost of ramping could degrade the value of the RES. Hence, there is value for curtailing (or spilling) some of the RES potential output (Lamadrid and Mount, 2012). Particularly, consider two RES dispatch policies, full and partial dispatch. In the 
full dispatch policy, we inject all available renewable power to the grid, while in the partial dispatch policy, we inject only a fraction of the available renewable power to the grid in order to reduce ramping by the conventional generators. However, the choice between these two policies is not clear.

At first glance, the full dispatch policy seems to dominate the partial renewable dispatch policy, due to its potential to reduce the total operating cost in an economic merit order, thanks to the low marginal generation cost of RES. However, the uncertain RES and its variability require ramping by the conventional generators, thus it might be less costly to dispatch renewable power partially. To illustrate this effect, consider a simplified system where there are no power flow and capacity constraints, and there is only one conventional generator and one RES generator. Given the dispatch policy in period $t-1$, and the observed renewable power in time periods $t-1$ and $t$, we want to find the dispatch policy in period $t$ in order to minimize the total cost. Let $D$ be the constant demand; thus we have the following optimization problem:

$$
\begin{array}{ll}
\min & C_{p}\left(p^{t}\right)+C_{r}\left(r^{t}\right) \\
\text { s.t. } & \\
& p^{t}+y^{t}=D \\
& p^{t}-p^{t-1}-r^{t}=0 \\
& 0 \leq y^{t} \leq w^{t}
\end{array}
$$

In this model, $p^{t}$ and $r^{t}$ are decision variables respectively representing the power generated (MWh) by the conventional generator and the amount of ramping up/down (MWh) in time period $t$. The decision variable $y^{t}$ is the amount of power injected by the renewable generator to the system at time $t$. Additionally, $p^{t-1}=D-y^{t-1}$ represents the dispatch policy of period $t-1$ and is given. By considering linear generation costs

$$
C_{p}\left(p^{t}\right)=c_{p} p^{t}
$$

for some constant $c_{p}$, and quadratic ramping costs

$$
C_{r}\left(r^{t}\right)=c_{r}\left(r^{t}\right)^{2}
$$

for some constant $c_{r}$, one can write the model as:

$$
\begin{aligned}
& \min c_{p} D-c_{p} y^{t}+\frac{1}{2} c_{r}\left(y^{t-1}-y^{t}\right)^{2} \\
& \text { s.t. } 0 \leq y^{t} \leq w^{t}
\end{aligned}
$$

Given $y^{t-1}$, the optimal value for $y^{t}$ is:

$$
y_{*}^{t}= \begin{cases}\frac{c_{p}}{c_{r}}+y^{t-1}, & \text { if } w^{t} \geq \frac{c_{p}}{c_{r}}+y^{t-1} \\ w^{t}, & \text { otherwise. }\end{cases}
$$

Define $w^{t}-y^{t-1}$ as the maximum available ramp capacity in period $t$. A system operator whose objective is to minimize cost would rather implement the full renewable dispatch if the marginal ramping costs at the maximum available ramp capacity is less than the marginal generation cost, that is, when $c_{p} \geq c_{r}\left(w^{t}-y^{t-1}\right)$, as shown in Figure 1(a). Moreover, the system operator prefers partial renewable dispatch if the marginal ramping costs at the maximum available ramp capacity is greater than the marginal generation cost, that is when $c_{p} \leq c_{r}\left(w^{t}-y^{t-1}\right)$, as shown in Figure 1(b). The numerical results presented in Section 5 show that this behavior is also evident in more realistic instances of the ED problem. 


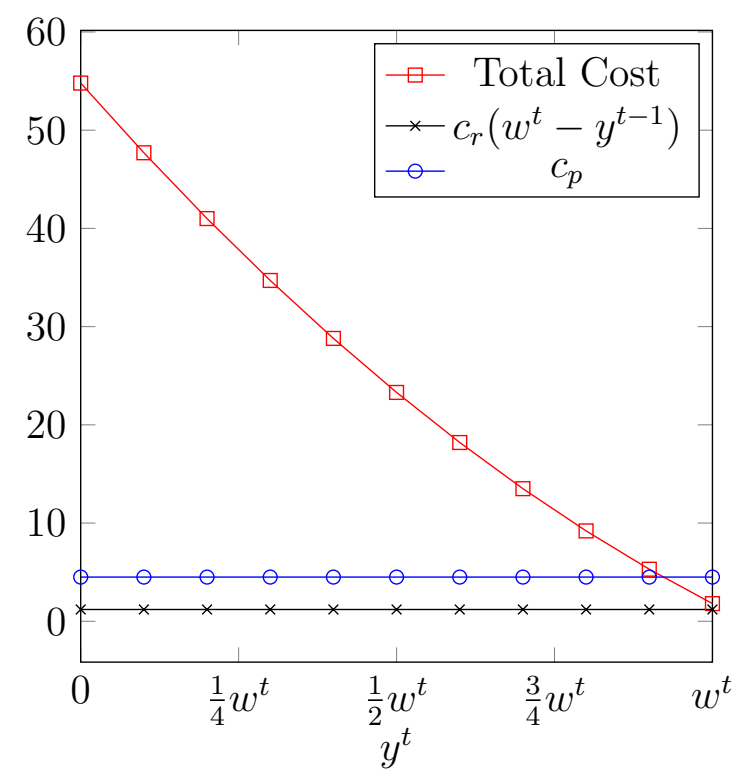

(a) Full Dispatch

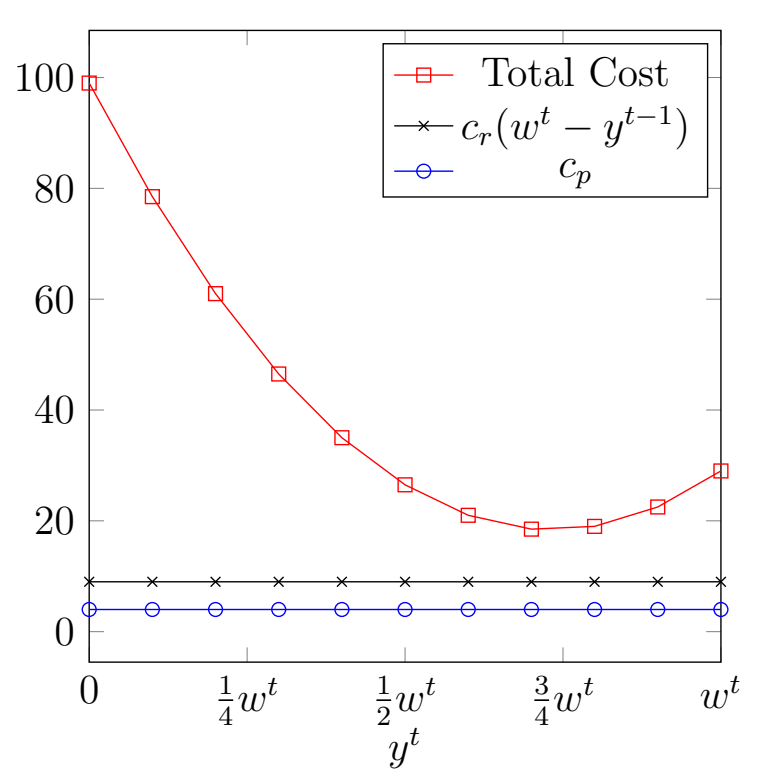

(b) Partial Dispatch

Figure 1: Renewable dispatch policy

\section{Deterministic Formulation}

As discussed earlier, ramping costs are important and have significant effects on the generation and dispatch policies in the power network. These effects need to be quantified and addressed properly to have a reliable dispatch with minimum cost. For this purpose, we begin by developing a mathematical Direct Current (DC) approximation of the optimal power flow model with ramping costs and constraints in a deterministic setting. Then, in Section 4 we extend it to a chance constrained optimization model to obtain a reliable dispatch policy when there are variable and uncertain RES in the network.

Consider a network of $n$ buses containing a set $\mathcal{G}$ of generator buses and a set $\mathcal{D}$ of demand (load) buses. That is, $\mathcal{N}=\mathcal{G} \cup \mathcal{D}$, with $|\mathcal{N}|=n$. These sets are connected by transmission branches defined in set $\mathcal{L}$. Also, $\boldsymbol{Y} \in \mathcal{C}^{n \times n}$ is the admittance matrix, and $\boldsymbol{B}$ is the corresponding susceptance matrix. Let $\theta_{i}^{t}$ and $V_{i}^{t}$ be the voltage phase angle and voltage amplitude of bus $i \in \mathcal{N}$ at time $t=1, \ldots, T$, where $T$ is the optimization horizon. Then, the DC power flow equations for time $t$ are:

$$
\begin{array}{lr}
V_{i}^{t} V_{j}^{t} B_{i j}\left(\theta_{i}^{t}-\theta_{j}^{t}\right) \leq \bar{f}_{i j} \quad \forall i \neq j \in \mathcal{N} \\
\sum_{j \in \mathcal{N}} V_{i}^{t} V_{j}^{t} B_{i j}\left(\theta_{i}^{t}-\theta_{j}^{t}\right)=q_{i}^{t} & \forall i \in \mathcal{N} .
\end{array}
$$

where $q_{i}^{t}$ is the real power injection of node $i \in \mathcal{N}$ at time $t$ and $\bar{f}_{i j}$ is the thermal capacity limit on the branch connecting nodes $(i, j) \in \mathcal{N}$. Note that the net power injection for a generator bus is positive and for a load bus is negative, i.e.,

$$
\begin{cases}q_{i}^{t} \geq 0 & i \in \mathcal{G} \\ q_{i}^{t} \leq 0 & i \in \mathcal{D} .\end{cases}
$$

Under the connectedness assumption for the underlying graph, there exists a feasible vector $\boldsymbol{\theta}^{t}$ of 
voltage angles in period $t$ for the the system (10), if and only if $\sum_{i \in \mathcal{N}} q_{i}^{t}=0$ for $t=1,2, . ., T$ (Bienstock et al., 2012, Lemma 1.1).

Defining a set of new variables ${ }^{1} \delta_{i j}^{t}=\theta_{i}^{t}-\theta_{j}^{t}$ to be the voltage angle difference from bus $i$ to bus $j$ and new parameters $\omega_{i j}^{t}=V_{i}^{t} V_{j}^{t} B_{i j}$, one can rewrite system (10) as the following system of linear inequalities (the details of the derivation can be found in Moarefdoost and Snyder (2015) and Bienstock et al. (2012)):

$$
\begin{aligned}
\hat{\boldsymbol{B}} \hat{\boldsymbol{q}}^{t} & \leq \overline{\boldsymbol{f}} \\
-\hat{\boldsymbol{B}} \hat{\boldsymbol{q}}^{t} & \leq \overline{\boldsymbol{f}},
\end{aligned}
$$

where $\hat{\boldsymbol{B}}$ is a special $|\mathcal{L}| \times(n-1)$-matrix, $\overline{\boldsymbol{f}}$ is an $|\mathcal{L}|$-vector of line capacity limits, and $\hat{\boldsymbol{q}}^{t}$ is an $(n-1)$-vector of net power export from each node, excluding the reference bus.

Let $p_{i}^{t}$ be the power generated by generator $i \in \mathcal{G}$ and $r_{i}^{t}$ be its power ramp up/down rate (generator $i$ is ramping up when $r_{i}^{t} \geq 0$, and ramping down otherwise) at time $t=1,2, . ., T$, and $\hat{q}_{i}^{t}=-d_{i}^{t}, \forall i \in \mathcal{D}$, and $\hat{q}_{i}^{t}=p_{i}^{t}, \forall i \in \mathcal{G}$, where $d_{i}^{t}$ is the load of bus $i$ at time $t$. Without loss of generality, assume that the rows of $\hat{\boldsymbol{q}}^{t}$ are arranged in such a way that the first $|\mathcal{D}|$ rows of $\hat{\boldsymbol{q}}^{t}$ correspond to the load buses and the remaining rows correspond to the generators. Thus, we can partition $\hat{\boldsymbol{B}}$ into two matrices $\hat{\boldsymbol{B}}^{d}$ and $\hat{\boldsymbol{B}}^{g}$, where $\hat{\boldsymbol{B}}^{d}$ contains the columns of matrix $\hat{\boldsymbol{B}}$ corresponding to the load buses (or negative injections) and $\hat{\boldsymbol{B}}^{g}$ contains the columns of matrix $\hat{\boldsymbol{B}}$ corresponding to the generator buses. Finally, for $t=1,2, . ., T, \forall i \in \mathcal{G}$ and $\forall l \in \mathcal{L}$ we can write the mathematical model as:

$$
\begin{array}{ll}
\min \sum_{i \in \mathcal{G}} \sum_{t=1}^{T} C_{p}\left(p_{i}^{t}\right)+C_{r}\left(r_{i}^{t}\right) & \\
\text { s.t. } & \\
\sum_{i \in \mathcal{D}} d_{i}^{t}-\sum_{i \in \mathcal{G}} p_{i}^{t}=0 & \forall t \\
\sum_{i \in \mathcal{G}} \hat{B}_{l i}^{g} p_{i}^{t} \leq \bar{f}_{l}+\sum_{i \in \mathcal{D}} \hat{B}_{l i}^{d} d_{i}^{t} & \forall l \in \mathcal{L}, \forall t \\
-\sum_{i \in \mathcal{G}} \hat{B}_{l i}^{g} p_{i}^{t} \leq \bar{f}_{l}-\sum_{i \in \mathcal{D}} \hat{B}_{l i}^{d} d_{i}^{t} & \forall l \in \mathcal{L}, \forall t \\
p_{i}^{t}=p_{i}^{t-1}+r_{i}^{t} \tau & \forall i \in \mathcal{G}, \forall t \\
P_{i}^{\text {min }} \leq p_{i}^{t} \leq P_{i}^{\text {max }} & \forall i \in \mathcal{G}, \forall t \\
r_{i}^{\text {min }} \leq r_{i}^{t} \tau \leq r_{i}^{\text {max }} & \forall i \in \mathcal{G}, \forall t .
\end{array}
$$

In this model, $p_{i}^{t}$ and $r_{i}^{t}$ are decision variables $\forall i \in \mathcal{G}$ and $\tau$ is the elapsed time between time periods $t$ and $t+1$ for $t=1,2, \cdots, T$. The objective cost function (13) consists of the power generation cost, $C_{p}(\cdot)$ and the ramping cost, $C_{r}(\cdot)$. Here, we consider $C_{p}(\cdot)$ to be a linear function of the power generation level, see eq. (5), and the ramping cost $C_{r}(\cdot)$ to be a quadratic function of the ramp rate, see eq. (6). This is consistent with the literature in which energy costs are either piecewise linear or quadratic (Cain et al., 2012b; Lamadrid et al., 2014; Ela and O'Malley, 2012; Moarefdoost and Snyder, 2015); and ramping costs are piecewise linear or quadratic (Tanaka, 2006;

\footnotetext{
${ }^{1}$ This is a standard way to model the angle variables in OPF problems. See Cain et al. (2012a)
} 
Sherestha et al., 2004; Wang and Shahidehpour, 1995). Moreover, this ensures that the resulting problem has a piece-wise convex objective function. This feature of the objective function allows both this deterministic formulation, (13)-(19), as well as the stochastic models that we study later to be solved to optimality using current solvers.

Constraints $(15)$ and $(16)$ are the DC power flow constraints on each branch $l \in \mathcal{L}$. In constraints (14), $d_{i}^{t}$ is the demand of bus $i \in \mathcal{D}$ in time period $t$. These constraints are the power balance equations, ensuring that the total power demand equals the total power supply at each time period. Constraints (17) are the ramping inter-temporal constraints for the active power. Finally, the lower bounds and upper bounds on the decision variables are defined by constraints (18) and (19). Notice that we have initial conditions on $p_{i}^{0}=p_{0 i}$ for $i \in \mathcal{G}$.

\section{Stochastic Formulation}

Assume that the set of generator buses $\mathcal{G}$ contains a set of conventional generators $\overline{\mathcal{G}}$ and a set of non-dispatchable RES generators $\tilde{\mathcal{G}}$. Let $\tilde{p}_{j}^{t}$ be a random variable representing the output of the RES generator $j \in \tilde{\mathcal{G}}$ in time period $t$ and $x_{j}^{t} \in[0,1]$ be the fraction of its power dispatched at time $t$ by the system operator. In order to account for the randomness of $\tilde{p}_{j}^{t}$ in the dispatch problem, we first reformulate the power balance equality constraints (20) so that they hold for any possible uncertain realizations of $\tilde{p}_{j}^{t}, \forall j \in \tilde{\mathcal{G}}$. We therefore rewrite the equality constraints (14) as:

$$
\sum_{i \in \mathcal{D}} d_{i}^{t}-\sum_{j \in \tilde{\mathcal{G}}} x_{j}^{t} \tilde{p}_{j}^{t}-\sum_{i \in \overline{\mathcal{G}}} p_{i}^{t}=0 \quad \forall t
$$

As stated in Bienstock et al. (2012), the dispatchable power output needs to be adjusted in real time in response to the uncertain output of the RES generators. This requires a secondary affine control to reset the output of conventional generators within the ramping period $\tau$ of analysis. We model the power output of a RES generator $j \in \tilde{\mathcal{G}}$ similar to Bienstock et al. (2012) as follows:

$$
\tilde{p}_{j}^{t}=\mu_{j}^{t}+\varepsilon_{j}^{t}
$$

where $\mu_{j}^{t}$ is the mean RES output and $\varepsilon_{j}^{t}$ is a zero mean random variable. Then, the affine control that resets the output of conventional generator $i \in \overline{\mathcal{G}}$ is given by:

$$
p_{i}^{t}=\bar{p}_{i}^{t}-\frac{1}{|\overline{\mathcal{G}}|} \sum_{j \in \tilde{\mathcal{G}}} x_{j}^{t} \varepsilon_{j}^{t} .
$$

By (21)-(22), the equality constraints (20) hold for any possible uncertain realization of $\tilde{p}_{j}^{t}, \forall j \in \tilde{\mathcal{G}}$ if

$$
\sum_{i \in \mathcal{D}} d_{i}^{t}-\sum_{j \in \tilde{\mathcal{G}}} x_{j}^{t} \mu_{j}^{t}-\sum_{i \in \overline{\mathcal{G}}} \bar{p}_{i}^{t}=0, \quad \forall t .
$$

Additionally, as a consequence of the affine control we have:

$$
r_{i}^{t}=\bar{r}_{i}^{t}-\frac{1}{\tau|\overline{\mathcal{G}}|} \sum_{j \in \tilde{\mathcal{G}}} x_{j}^{t} \varepsilon_{j}^{t}+\frac{1}{\tau|\overline{\mathcal{G}}|} \sum_{j \in \tilde{\mathcal{G}}} x_{j}^{t-1} \varepsilon_{j}^{t-1}
$$


thus, the ramping equality constraints (equation (17) in the deterministic problem formulation) hold if

$$
\bar{p}_{i}^{t}=\bar{p}_{i}^{t-1}+\bar{r}_{i}^{t} \tau, \quad \forall i \in \mathcal{G}, \forall t .
$$

After these modifications we have a reformulation in terms of the decision variable $\bar{p}, \bar{r} \in \mathcal{R}^{\mathcal{G}}$ and $x \in \mathcal{R}^{\mathcal{D}}$. Moreover, constraints (15), (16), (18), and (19) in the deterministic model need to be reformulated to take into account the RES uncertainty.

A widely accepted approach in the literature is to recast these constraints as chance constraints (cf., Birge and Louveaux, 1997), ensuring that the balance and flow constraints are satisfied with a certain probability. Specifically, let $\hat{\boldsymbol{B}}^{\bar{g}}$ be the matrix of columns of $\hat{\boldsymbol{B}}^{g}$ corresponding to the controllable generators, and $\hat{\boldsymbol{B}}^{\tilde{g}}$ be the matrix of columns of $\hat{\boldsymbol{B}}^{g}$ corresponding to the RES generators. The flow chance constraints (equations (15) and (16) in the deterministic problem formulation) are respectively formulated as:

$$
\begin{aligned}
& \operatorname{Prob}\left\{-\sum_{i \in \overline{\mathcal{G}}} \hat{B}_{l i}^{\bar{g}} p_{i}^{t}-\sum_{j \in \tilde{\mathcal{G}}} \hat{B}_{l j}^{\tilde{g}} x_{j}^{t} \tilde{p}_{j}^{t} \geq-\bar{f}_{l}-\sum_{i \in \mathcal{D}} \hat{B}_{l i}^{d} d_{i}^{t}\right\} \geq 1-\gamma_{r}, \quad \forall l \in \mathcal{L}, t=1,2 \cdots, T, \\
& \operatorname{Prob}\left\{\sum_{i \in \overline{\mathcal{G}}} \hat{B}_{l i}^{\bar{g}} p_{i}^{t}+\sum_{j \in \tilde{\mathcal{G}}} \hat{B}_{l j}^{\tilde{g}} x_{j}^{t} \tilde{p}_{j}^{t} \geq-\bar{f}_{l}+\sum_{i \in \mathcal{D}} \hat{B}_{l i}^{d} d_{i}^{t}\right\} \geq 1-\gamma_{l}, \quad \forall l \in \mathcal{L}, t=1,2 \cdots, T,
\end{aligned}
$$

where $\tilde{p}_{j}^{t}$ and $p_{i}^{t}$ are defined as in (21) and (22), respectively. Similarly, the power limit constraints (equation (18) in the deterministic problem formulation) are respectively formulated as:

$$
\begin{array}{ll}
\operatorname{Prob}\left\{-\bar{p}_{i}^{t}+\frac{1}{|\overline{\mathcal{G}}|} \sum_{j \in \tilde{\mathcal{G}}} x_{j}^{t} \varepsilon_{j}^{t} \geq-P_{i}^{\text {max }}\right\} \geq 1-\gamma_{r}, \quad & \forall i \in \overline{\mathcal{G}}, t=1,2 \cdots, T, \\
\operatorname{Prob}\left\{\bar{p}_{i}^{t}-\frac{1}{|\overline{\mathcal{G}}|} \sum_{j \in \tilde{\mathcal{G}}} x_{j}^{t} \varepsilon_{j}^{t} \geq P_{i}^{\text {min }}\right\} \geq 1-\gamma_{l}, & \forall i \in \overline{\mathcal{G}}, t=1,2 \cdots, T .
\end{array}
$$

Finally, the ramping limit constraints (equation (19) in the deterministic problem formulation) are respectively formulated as:

$$
\begin{aligned}
& \operatorname{Prob}\left\{-\bar{r}_{i}^{t} \tau+\frac{1}{|\overline{\mathcal{G}}|} \sum_{j \in \tilde{\mathcal{G}}} x_{j}^{t} \varepsilon_{j}^{t}-\frac{1}{|\overline{\mathcal{G}}|} \sum_{j \in \tilde{\mathcal{G}}} x_{j}^{t-1} \varepsilon_{j}^{t-1} \geq-r_{i}^{m a x}\right\} \geq 1-\gamma_{r}, \quad \forall i \in \overline{\mathcal{G}}, t=1,2 \cdots, T, \\
& \operatorname{Prob}\left\{\bar{r}_{i}^{t} \tau-\frac{1}{|\overline{\mathcal{G}}|} \sum_{j \in \tilde{\mathcal{G}}} x_{j}^{t} \varepsilon_{j}^{t}+\frac{1}{|\overline{\mathcal{G}}|} \sum_{j \in \tilde{\mathcal{G}}} x_{j}^{t-1} \varepsilon_{j}^{t-1} \geq r_{i}^{m i n}\right\} \geq 1-\gamma_{l}, \quad \forall i \in \overline{\mathcal{G}}, t=1,2 \cdots, T .
\end{aligned}
$$

In equations (26)-(31), small values of $\gamma_{r}$ and $\gamma_{l}$ imply that the probability of satisfaction of the underlying constraint is high. While all values of $\gamma_{r}$ and $\gamma_{l}$ could be different, for simplicity, we define them to a single value $\gamma_{r}$ for the right inequalities and $\gamma_{l}$ for the left inequalities.

Next, we convert the probabilistic constraints (26), (27) (28), (29), (30) and (31) into their deterministic equivalent formulation (cf., Birge and Louveaux, 1997). In general, this requires to 
reformulate probabilistic constraints of the form

$$
\operatorname{Prob}\{G(x, \boldsymbol{\xi}) \geq R\} \geq 1-\gamma
$$

where $x$ is a decision variable, $\boldsymbol{\xi}$ is a random variable, and $R$ and $\gamma$ are appropriate constants. A generally accepted approach to do so is by sampling a number of realizations (scenarios) for $\boldsymbol{\xi}$, to approximate $\operatorname{Prob}\{G(x, \boldsymbol{\xi}) \geq R\}$ and write the resulting optimization problem as a mixed-integer program (MIP, Luedtke et al. (2007); Ahmed and Shapiro (2008); Luedtke (2010)). ${ }^{2}$

Alternatively, if the probability distribution of $G(x, \boldsymbol{\xi})$ is known, the $\operatorname{Prob}\{G(x, \boldsymbol{\xi}) \geq R\}$ can be computed explicitly (see, e.g., Filomena and Lejeune, 2014; Bonami and Lejeune, 2009; Charnes and Cooper, 1963). To illustrate this second approach, consider the following probabilistic constraint:

$$
\operatorname{Prob}\left\{\boldsymbol{\xi}^{T} x \geq R\right\} \geq 1-\gamma
$$

where $\boldsymbol{\xi}$ is a random vector with mean $\boldsymbol{\mu}$ and variance-covariance matrix $\boldsymbol{\Sigma}$. The deterministic equivalent of (32) is the following nonlinear constraint (Bonami and Lejeune, 2009):

$$
\boldsymbol{\mu}^{T} x+F_{(x)}^{-1}(\gamma) \sqrt{x^{T} \Sigma x} \geq R
$$

where $F_{(x)}(\psi)$ is the cumulative probability distribution of the following random variable:

$$
\psi=\frac{\boldsymbol{\mu}^{T} x-\boldsymbol{\xi}^{T} x}{\sqrt{x^{T} \Sigma x}},
$$

and $F_{(x)}^{-1}(\alpha)$ is its inverse. Bonami and Lejeune (2009) show that when $0<\gamma \leq 0.5$ and the probability distribution of $\boldsymbol{\xi}^{T} x$ is symmetric or positively skewed, the deterministic equivalent (33) of the probabilistic constraint (32) is a second-order cone constraint (cf., Boyd and Vandenberghe, 2009). Note that low values of $\gamma$ in (32) correspond to a chance constraint in which the probability of the constraint being satisfied is high. As described in Bonami and Lejeune (2009), the main advantage of reformulating a chance constraint as a second-order cone constraint is that the resulting optimization problem (that is referred as a second-order cone program - SOCP) is convex and can then be solved efficiently using interior-point optimization methods (cf., Boyd and Vandenberghe, 2009). Bienstock et al. (2012) take advantage of this fact in order to efficiently model the demand uncertainy in an economic dispatch problem with network constraints when the demand is assumed to follow a normal distribution. Next, we show that a similar approach can be used to address the non-normal uncertainty of RES power output in dispatch problems.

\subsection{Robust Formulation}

As stated earlier, it is possible to write the deterministic equivalent of a chance constraint using the probability distribution of the source of uncertainty. Now, assume that we have the probability distribution of the random variables $\varepsilon_{j}^{t}$ for $t=1,2, . ., T$ and $\forall j \in \tilde{\mathcal{G}}$ and their affine combinations. Thus, one might compute the probability constraints (26), (27), (28), (29),(30) and (31) explicitly. Specifically, assume that we are given the probability distribution of $\sum_{j \in \tilde{\mathcal{G}}} a_{j} \varepsilon_{j}^{t}, \forall t$ and $F_{a}(\cdot)$ is

\footnotetext{
${ }^{2}$ Appendix A provides the details of the MIP formulation of the problem as a reference to the reader.
} 
its cumulative probability distribution $\left(F_{a}^{-1}(\cdot)\right.$ is its inverse). Also, $\forall i, j \in \tilde{\mathcal{G}}$ and $\forall t, E\left(\varepsilon_{j}^{t}\right)=0$, $\operatorname{cov}\left(\varepsilon_{i}^{t}, \varepsilon_{j}^{t}\right)=\sigma_{i j}$, and $\operatorname{cov}\left(\varepsilon_{j}^{t}, \varepsilon_{j}^{t-1}\right)=0$. Now, let us define the following parameters:

$$
\begin{array}{rlrl}
D_{l}^{t} & =\sum_{i \in \mathcal{D}} \hat{B}_{l i}^{d} d_{i}^{t}, & & \forall l \in \mathcal{L}, \forall t \\
\bar{\beta}_{l} & =\frac{1}{|\overline{\mathcal{G}}|} \sum_{i \in \overline{\mathcal{G}}} \hat{B}_{l i}^{\bar{g}}, & & \forall l \in \mathcal{L} \\
\xi_{j l}^{t}=\mu_{j}^{t} \hat{B}_{l j}^{\tilde{g}}, & & \forall j \in \tilde{\mathcal{G}}, \forall l \in \mathcal{L}, \forall t \\
\sigma_{i j}^{l}=\left(\hat{B}_{l i}^{\tilde{g}}-\bar{\beta}_{l}\right)\left(\hat{B}_{l j}^{\tilde{g}}-\bar{\beta}_{l}\right) \sigma_{i j}, & & \forall i, j \in \tilde{\mathcal{G}}, \forall l \in \mathcal{L} .
\end{array}
$$

Then the deterministic equivalent of the stochastic economic dispatch problem with network constraints can be formulated as follows:

$$
\begin{array}{ll}
\min _{\sum_{i \in \overline{\mathcal{G}}}} \sum_{t=1}^{T} E_{\boldsymbol{w}}\left[C_{p}\left(\bar{p}_{i}^{t}-\frac{1}{|\overline{\mathcal{G}}|} \sum_{j \in \tilde{\mathcal{G}}} x_{j}^{t} \varepsilon_{j}^{t}\right)+C_{r}\left(\bar{r}_{i}-\frac{1}{|\overline{\mathcal{G}}| \tau} \sum_{j \in \tilde{\mathcal{G}}} x_{j}^{t} \varepsilon_{j}^{t}-x_{j}^{t-1} \varepsilon_{j}^{t-1}\right)\right] & \\
\text { s.t. } & \forall t \\
\sum_{i \in \mathcal{D}} d_{i}^{t}-\sum_{j \in \tilde{\mathcal{G}}} \mu_{j}^{t}-\sum_{i \in \overline{\mathcal{G}}} \bar{p}_{i}^{t}=0 & \forall t, l \in \mathcal{L} \\
-\sum_{i \in \overline{\mathcal{G}}} \hat{B}_{l i}^{\bar{g}} \bar{p}_{i}^{t}-\sum_{j \in \tilde{\mathcal{G}}} \xi_{j l}^{t} x_{j}^{t}+D_{l}^{t}+\bar{f}_{l}+F_{(x)}^{-1}\left(\gamma_{r}\right) \sqrt{\sum_{i \in \tilde{\mathcal{G}}} \sum_{j \in \tilde{\mathcal{G}}} \sigma_{i j}^{l} x_{i}^{t} x_{j}^{t}} \geq 0 & \forall t, l \in \mathcal{L} \\
\sum_{i \in \overline{\mathcal{G}}} \hat{B}_{l i}^{\bar{g}} \bar{p}_{i}^{t}+\sum_{j \in \tilde{\mathcal{G}}} \xi_{j l}^{t} x_{j}^{t}-D_{l}^{t}+\bar{f}_{l}+F_{(x)}^{-1}\left(\gamma_{l}\right) \sqrt{\sum_{i \in \tilde{\mathcal{G}}} \sum_{j \in \tilde{\mathcal{G}}} \sigma_{i j}^{l} x_{i}^{t} x_{j}^{t}} \geq 0 & \forall t, k \in \overline{\mathcal{G}} \\
\bar{r}_{k}^{t} \tau|\overline{\mathcal{G}}|-r_{k}^{\min }|\overline{\mathcal{G}}|+F_{(x)}^{-1}\left(\gamma_{l}\right) \sqrt{\sum_{i \in \tilde{\mathcal{G}}} \sum_{j \in \tilde{\mathcal{G}}} \sigma_{i j}\left(x_{i}^{t} x_{j}^{t}+x_{i}^{t-1} x_{j}^{t-1}\right)} \geq 0 & \forall t, k \in \overline{\mathcal{G}} \\
-\bar{r}_{k}^{t} \tau|\overline{\mathcal{G}}|+r_{k}^{\max }|\overline{\mathcal{G}}|+F_{(x)}^{-1}\left(\gamma_{r}\right) \sqrt{\sum_{i \in \tilde{\mathcal{G}}} \sum_{j \in \tilde{\mathcal{G}}} \sigma_{i j}\left(x_{i}^{t} x_{j}^{t}+x_{i}^{t-1} x_{j}^{t-1}\right)} \geq 0 & \forall t, k \in \overline{\mathcal{G}} \\
\bar{p}_{k}^{t}|\overline{\mathcal{G}}|-p_{k}^{\min }|\overline{\mathcal{G}}|+F_{(x)}^{-1}\left(\gamma_{l}\right) \sqrt{\sum_{i \in \tilde{\mathcal{G}}} \sum_{j \in \tilde{\mathcal{G}}} \sigma_{i j} x_{i}^{t} x_{j}^{t}} \geq 0 & \forall t, k \in \overline{\mathcal{G}} \\
-\bar{p}_{k}^{t}|\overline{\mathcal{G}}|+p_{k}^{\max }|\overline{\mathcal{G}}|+F_{(x)}^{-1}\left(\gamma_{r}\right) \sqrt{\sum_{i \in \tilde{\mathcal{G}}} \sum_{j \in \tilde{\mathcal{G}}} \sigma_{i j} x_{i}^{t} x_{j}^{t}} \geq 0 & \forall t, k \in \overline{\mathcal{G}} \\
\bar{p}_{k}^{t}=\bar{p}_{k}^{t-1}+\bar{r}_{i}^{k} \tau & \forall t, k \in \tilde{\mathcal{G}} \\
0 \leq x_{k}^{t} \leq 1 &
\end{array}
$$

The objective function (34) for the case of linear generation cost and quadratic ramping cost, see eqs. (5) and (6), has the following form:

$$
\sum_{t=1}^{T} \sum_{i \in \overline{\mathcal{G}}} c_{p}^{i} \bar{p}_{i}^{t}+\frac{1}{2} c_{r}^{i}\left(\bar{r}_{i}^{t}\right)^{2}+\frac{1}{|\overline{\mathcal{G}}| \tau^{2}} \sum_{t=1}^{T} \sum_{i \in \overline{\mathcal{G}}} \sum_{j, k \in \tilde{\mathcal{G}}} c_{r}^{i} \sigma_{j k}\left(x_{j}^{t} x_{k}^{t}+x_{j}^{t-1} x_{k}^{t-1}\right) .
$$


Note that the matrix of $\sigma_{i j}$, for all $i, j \in \tilde{\mathcal{G}}$ is positive semidefinite. Similarly, for all $l \in \mathcal{L}$, the matrices of $\sigma_{i j}^{l}$ for all $i, j \in \tilde{\mathcal{G}}$, are positive semidefinite (cf., Boyd and Vandenberghe, 2009). Therefore, the objective (44) is convex, and the constraints (35)-(43) are well defined. In order to have a convex optimization model, $F_{(x)}^{-1}(\cdot)$ should be independent of the decision variables, $x_{i}, \forall i \in \tilde{\mathcal{G}}$, and be non-positive, that is $F_{(x)}^{-1}(\cdot) \leq 0$. In fact, first, we need to find conditions under which $F_{(x)}^{-1}(\cdot) \leq 0$. Second, we need to obtain a good approximation for $F_{(x)}^{-1}(\cdot)$ that is independent of $x_{i}, \forall i \in \tilde{\mathcal{G}}$.

Next, we focus on the case in which the uncertainty in the system is driven by wind energy sources. In this case, we take advantage of the well known behavior of the wind resource, particularly, the properties of the wind probability distribution.

Despite numerous theoretical and empirical studies on wind speed probability distribution (Hennessey and Joseph, 1977; Justus et al., 1978; Tuller and Brett, 1984; Garcia et al., 1998; Seguro and Lambert, 2000; Dorvlo, 2002; Barbounis et al., 2006; Morgan et al., 2011), there are few works that address specifically wind power probability distributions (Hetzer et al., 2008; Zuwei and Tuzuner, 2009; Louie and Sloughter, 2014). Previous studies in the literature (Tuller and Brett, 1984; Morgan et al., 2011; Masters, 2013) show that the wind speed profile at a given location can be fitted closely by a Weibull or a Rayleigh distribution over time.

A wind farm consists of multiple wind turbines (Norgaard and Hottlinen, 2004). The probability distribution of the wind farm's power output is the same as the probability distribution of the power output of a wind turbine with different mean and variance, assuming that all wind turbines in the farm are identical and experience almost the same wind speed. For an idealized wind turbine, the wind power as function of a given wind speed is (Louie and Sloughter, 2014):

$$
P_{w}(v)= \begin{cases}0 & v<v_{i} \\ \frac{v^{3}-v_{i}^{3}}{v_{r}^{3}-v_{i}^{3}} P_{\max } & v_{i} \leq v \leq v_{r} \\ P_{\max } & v_{r} \leq v \leq v_{o} \\ 0 & v>v_{o}\end{cases}
$$

where $P_{w}$ is the wind power output ( $\mathrm{kW}$ or MW), $P_{\max }$ is the rated wind power (KW or MW), $v_{i}$ is the cut-in wind speed (miles per hour), $v_{r}$ is the rated wind speed (miles per hour) and $v_{o}$ is the cut-out wind speed (miles per hour).

It is clear that the wind power has a mixed discrete-continuous probability distribution. Let $f_{V}, F_{V}$ be the probability distribution function (pdf) and cumulative distribution function (cdf) of the wind speed random variable, respectively, and let $f_{P_{w}}$ and $F_{P_{w}}$ be the pdf and cdf of the wind power random variable, respectively. Then we have:

$$
f_{P_{w}}(p)= \begin{cases}\alpha_{0}=F_{V}\left(v_{i}\right)+1-F_{V}\left(v_{o}\right), & p=0 \\ \frac{d F_{V}(v(p))}{d v} \cdot \frac{d v(p)}{d p}, & 0<p<P_{\max } \\ \alpha_{\max }=F_{V}\left(v_{o}\right)-F_{V}\left(v_{r}\right), & p=P_{\max }\end{cases}
$$

where $v(p)=\left(\frac{p\left(v_{r}^{3}-v_{i}^{3}\right)+P_{\max }^{2}}{v_{i}^{3}}\right)^{\frac{1}{3}}$.

In the SOCP formulation of $(34)-(43), F_{(x)}^{-1}(\gamma)$ is non-positive if the wind power probability distribution is positively skewed for $0<\gamma \leq 0.5$, or $F_{p_{w}}^{-1}(\gamma) \leq 0$ in a certain rage of $\gamma$. 
Lemma 1. The wind probability distribution defined in (46) is positively skewed if

$$
F_{V}^{-1}(\tilde{\alpha}) \leq\left(\alpha_{\max } v_{r}^{3}+\alpha_{0} v_{i}^{3}+\min \left\{v_{i}^{3} f_{V}\left(v_{i}\right), v_{r}^{3} f_{V}\left(v_{r}\right)\right\}\right)^{\frac{1}{3}}
$$

where $\tilde{\alpha}=0.5+F_{V}\left(v_{i}\right)-\alpha_{0}$.

Proof. 1.A probability distribution of a random variable is positively skewed if its median is smaller than its mean. The mean for the wind power probability distribution (46) is:

$$
\begin{aligned}
\mu_{P_{w}} & =E(P)=P_{\max } \alpha_{\max }+\int_{v_{i}}^{v_{r}}\left(\frac{v^{3}-v_{i}^{3}}{v_{r}^{3}-v_{i}^{3}} P_{\max }\right) f_{V}(v) d v \\
& =P_{\max }\left(\alpha_{\max }-F_{V}\left(v_{r}\right) \frac{v_{i}^{3}}{v_{r}^{3}-v_{i}^{3}}+F_{V}\left(v_{i}\right) \frac{v_{i}^{3}}{v_{r}^{3}-v_{i}^{3}}+\int_{v_{i}}^{v_{r}} \frac{v^{3}}{v_{r}^{3}-v_{i}^{3}} f_{V}(v) d v\right) .
\end{aligned}
$$

We can show that

$$
\mu_{P_{w}} \geq P_{\max }\left(\alpha_{\max }-F_{V}\left(v_{r}\right) \frac{v_{i}^{3}}{v_{r}^{3}-v_{i}^{3}}+F_{V}\left(v_{i}\right) \frac{v_{i}^{3}}{v_{r}^{3}-v_{i}^{3}}+\min \left\{\frac{v_{i}^{3}}{v_{r}^{3}-v_{i}^{3}} f_{V}\left(v_{i}\right), \frac{v_{r}^{3}}{v_{r}^{3}-v_{i}^{3}} f_{V}\left(v_{r}\right)\right\}\right) .
$$

Let $P_{m}$ be the median of the wind power distribution. Then

$$
\operatorname{Prob}\left\{p \leq P_{m}\right\}=0.5 \Leftrightarrow \operatorname{Prob}\{v \leq \tilde{V}\}+\alpha_{0}=0.5 \Leftrightarrow F_{V}(\tilde{V})=0.5+F_{V}\left(v_{i}\right)-\alpha_{0} .
$$

Let $\tilde{\alpha}=0.5+F_{V}\left(v_{i}\right)-\alpha_{0}$. Then $\tilde{V}=F_{V}^{-1}(\tilde{\alpha})$ and $P_{m}=\frac{\tilde{V}^{3}-v_{i}^{3}}{v_{r}^{3}-v_{i}^{3}} P_{\max }$. The wind power distribution has positive skewness if $P_{m} \leq \mu_{P_{w}}$, and this is always true if

$$
\begin{aligned}
F_{V}^{-1}(\tilde{\alpha}) & \leq\left(\alpha_{\max }\left(v_{r}^{3}-v_{i}^{3}\right)-F_{V}\left(v_{r}\right) v_{i}^{3}+F_{V}\left(v_{i}\right) v_{i}^{3}+\min \left\{v_{i}^{3} f_{V}\left(v_{i}\right), v_{r}^{3} f_{V}\left(v_{r}\right)\right\}+v_{i}^{3}\right)^{\frac{1}{3}} \\
& \leq\left(\alpha_{\max } v_{r}^{3}+\alpha_{0} v_{i}^{3}+\min \left\{v_{i}^{3} f_{V}\left(v_{i}\right), v_{r}^{3} f_{V}\left(v_{r}\right)\right\}\right)^{\frac{1}{3}} .
\end{aligned}
$$

Therefore, given (47), for a single wind farm, $F_{(x)}^{-1}(\gamma) \leq 0$ when $0<\gamma \leq 0.5$. However, condition (47) is satisfied when the wind speed mean is close to the cut-in speed, $v_{i}$, which is not very common. To resolve this issue, one might relax the positive skewness condition by bounding the reliability probability, $\gamma$, in such a way that $F_{(x)}^{-1}(\gamma) \leq 0$.

Theorem 1. For a single wind farm, the deterministic equivalent of (32) for the case of uncertain wind power is a convex second-order cone if there exists $\gamma_{c} \in(0,1)$ such that $0<\gamma \leq \gamma_{c}$ and

$$
\gamma_{c} \leq \alpha_{0}+F_{V}\left(v_{i}\right)+F_{V}\left(\left(\alpha_{0} v_{i}^{3}+\alpha_{\max } v_{r}^{3}\right)^{\frac{1}{3}}\right)
$$

Proof. 1 Assume that there exists $\gamma_{c}$ such that $F_{P_{w}}^{-1}(\gamma) \leq 0$ for $0<\gamma \leq \gamma_{c}$. Then there exists $p_{c}$ such that $F_{P_{w}}\left(p_{c}\right)=\gamma_{c}$ and $p_{c}=P_{w}\left(v_{c}\right)$. Thus, we have $\gamma_{c}=\alpha_{0}+F_{V}\left(v_{c}\right)-F_{V}\left(v_{i}\right)$ and

$$
v_{c}=F_{V}^{-1}\left(\gamma_{c}-\alpha_{0}+F_{V}\left(v_{i}\right)\right) \text {. }
$$


In order to have $F_{P_{w}}^{-1}(\gamma) \leq 0$ for $0<\gamma \leq \gamma_{c}$, we need to have $p_{c} \leq \mu_{P_{w}}$, which implies

$$
\frac{v_{c}^{3}-v_{i}^{3}}{v_{r}^{3}-v_{i}^{3}} P_{\max } \leq P_{\max }\left(\alpha_{\max }-F_{V}\left(v_{r}\right) \frac{v_{i}^{3}}{v_{r}^{3}-v_{i}^{3}}+F_{V}\left(v_{i}\right) \frac{v_{i}^{3}}{v_{r}^{3}-v_{i}^{3}}+\int_{v_{i}}^{v_{r}} \frac{v^{3}}{v_{r}^{3}-v_{i}^{3}} f_{V}(v) d v\right) .
$$

With further algebraic simplification, we get

$$
v_{c} \leq\left(\alpha_{0} v_{i}^{3}+\alpha_{\max } v_{r}^{3}+\int_{v_{i}}^{v_{r}} v^{3} f_{V}(v) d v\right)^{\frac{1}{3}}
$$

This inequality holds if

$$
v_{c} \leq\left(\alpha_{0} v_{i}^{3}+\alpha_{\max } v_{r}^{3}\right)^{\frac{1}{3}}
$$

where $v_{c}=F_{V}^{-1}\left(\gamma_{c}-\alpha_{0}+F_{V}\left(v_{i}\right)\right)$. Therefore,

$$
\gamma_{c} \leq \alpha_{0}+F_{V}\left(v_{i}\right)+F_{V}\left(\left(\alpha_{0} v_{i}^{3}+\alpha_{\max } v_{r}^{3}\right)^{\frac{1}{3}}\right) .
$$

Theorem 1 provides conditions under which $F_{(x)}^{-1}(\gamma) \leq 0$ for a single wind farm. However, in a large scale problem, a power system may contain more than one wind farm. In that case, we are interested in the following random variable:

$$
\bar{P}_{(x)}=\sum_{i=1}^{N} x_{i} P_{w}^{i}
$$

where $N$ is the number of wind farms. If $N$ is sufficiently large and farms are distant from each other, $\bar{P}_{(x)}$ is approximately normal according to the law of large numbers and $F_{(x)}^{-1}(\gamma)$ can be easily obtained from a normal table. However, when $N$ is small, we need to approximate $F_{(x)}^{-1}(\gamma)$. For small enough $\gamma$, we have

$$
N \times \max \left\{F_{P_{w}^{i}}^{-1}(\gamma): i\right\} \leq F_{(x)}^{-1}(\gamma) \leq \max \left\{F_{P_{w}^{i}}^{-1}(\gamma): i\right\}
$$

We next show that we can use $N \times \max \left\{F_{P_{w}^{i}}^{-1}(\gamma): i\right\}$ as a proper approximation for $F_{(x)}^{-1}(\gamma)$. Given the wind power distribution, obtaining $N \max \left\{F_{P_{w}^{i}}^{-1}(\gamma): i\right\}$ is not a difficult task. Moreover, with this bound, a convex approximation of the problem formulated in (34)-(43) is obtained. In fact, using $N \times \max \left\{F_{P_{w}^{i}}^{-1}(\gamma): i\right\}$ results in a more conservative, robust solution. For the test problems, we replace $F_{(x)}^{-1}(\cdot)$ in equations (34)-(43) with the lower bound, $N \times \max \left\{F_{P_{w}^{i}}^{-1}(\cdot): i\right\}$.

To illustrate the accuracy of this approximation, we run a numerical simulation considering a Rayleigh probability distribution for the underlying wind speed. We consider wind farms with 10 wind turbines. We also assume all turbines in a farm are almost identical in terms of maximum rated power and technical characteristics. Figures $2(\mathrm{a})-2(\mathrm{~d})$ show the values of $N \times \max \left\{F_{P_{w}^{i}}^{-1}(\gamma)\right.$ : $i\}$ and $\max \left\{F_{P_{w}^{i}}^{-1}(\gamma): i\right\}$ for a power system with $N=2,3,4$ and $N=5$ wind farms. The farms have different power capacities that are chosen randomly between $9 \mathrm{MW}$ and $300 \mathrm{MW}$, similar to the minimum and maximum installed capacities of existing wind farms in the PJM system 
in $2010 .^{3}$ These figures illustrate that for realistic situations and for the small number of farms, approximation (52) is reasonable. The lower bound is not a tight bound for the cases where the number of farms is large. However, in that case, we can use the Normal quantile to obtain $F_{(x)}^{-1}(\gamma)$, thanks to the law of large numbers.

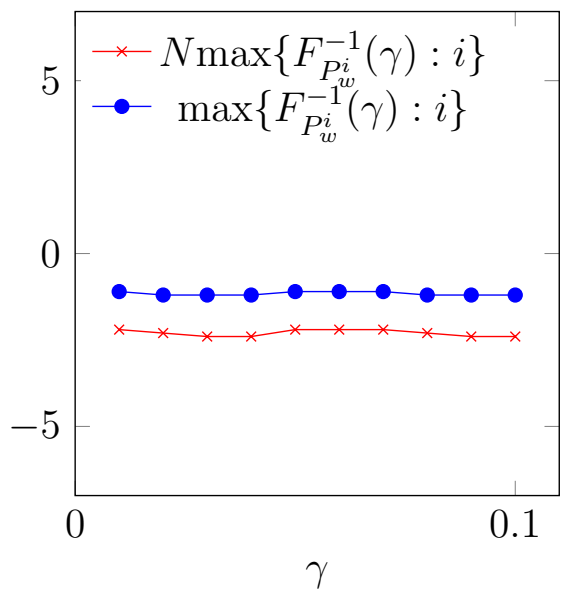

(a) $N=2$

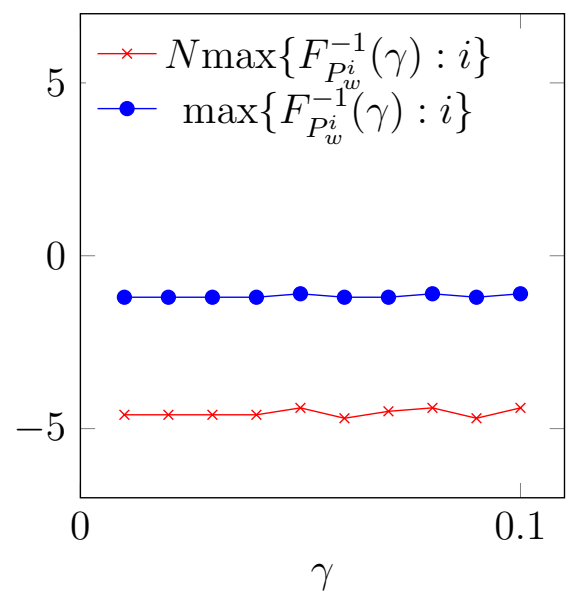

(c) $N=4$

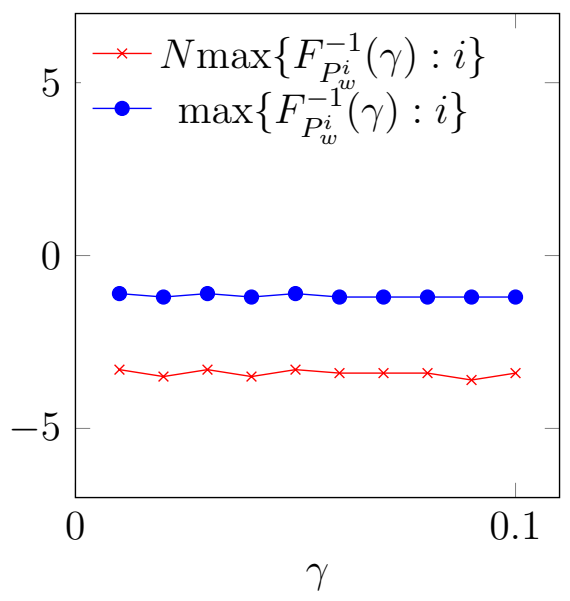

(b) $N=3$

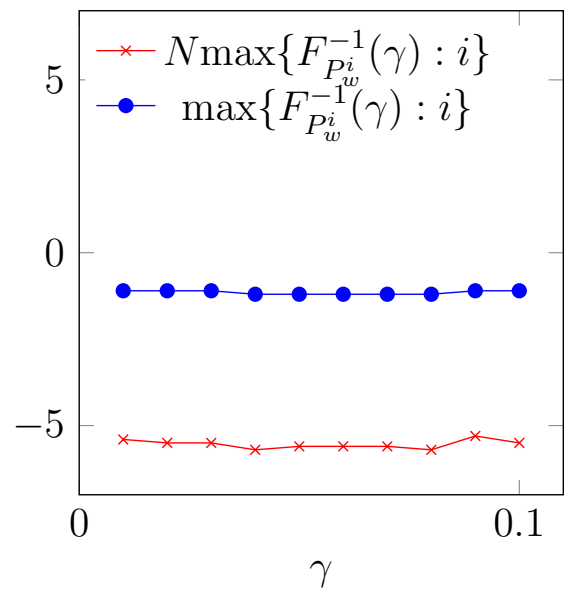

(d) $N=5$

Figure 2: Numerical Simulation for Quantile Approximation

\section{Numerical Analysis}

In Section 3, we develop methodological optimization models to minimize cost and maintain power system reliability. Here, we run a numerical experiment to study the performance of the proposed methodologies in detail. Overall, the results presented in this section provide evidence that our proposed method substantially decreases the solution time and provides solutions to instances where the sampling method does not in a reasonable time. We consider a 14-bus system

\footnotetext{
${ }^{3}$ PJM's EIA-411 report, www.pjm.com.
} 
with 2 wind farms and 3 conventional generators, a 30-bus system with 2 wind farms and 4 conventional generators, and a 118-bus system with 4 wind farms and 50 conventional generators. We run the experiment for $T=24$ hours with different levels for the variability in the mean of wind power output: low, medium and high. The variability is defined by the change in the mean of wind power between two consecutive periods. To measure the variability in the power output of wind generator $j \in \tilde{\mathcal{G}}$, we consider the following metric:

$$
\eta_{j}=\frac{\sum_{t=1}^{T-1}\left|\mu_{j}^{t}-\mu_{j}^{t+1}\right|}{\sum_{t=1}^{T} \mu_{j}^{t}} .
$$

The wind output has no variability when $\eta=0$, and it increases as $\eta$ increases. The value of $\eta$ for high, medium and low variability is $0.7,0.3$ and 0.1 , respectively. Since our focus is on the effect of wind variability and uncertainty on generation and ramping costs, we consider almost constant and flat demand profiles in order to isolate ramping due to load following from ramping due to the wind integration.

All numerical results for the three test networks are summarized in Tables 1-14, grouping the variables in three broad categories: financial variables (optimal cost, ramping costs); physical variables (total demand, renewable dispatch, conventional and renewable capacity); and the solution time.

For ramping, we consider two capability levels, 10\% and 30\%. The capability level is defined as the percentage of total capacity of a conventional generator that can be dedicated to ramping. We run experiments for reliability levels $1-\gamma$ of 0.95 and 0.98 for the 14 and 30 bus network. Also, we consider linear generation cost and quadratic ramping cost for generator $i$ as:

$$
c_{i}^{p} p+c_{i}^{r} r^{2}
$$

where, $p$ is generation quantity (MWh) and $r$ is the amount of ramping in MWh. The ramping cost coefficient is $13 \%$ of the generation cost coefficient, i.e, $c_{i}^{r}=0.13 c_{i}^{p}$. For $10 \%$ ramping capability, the observed cost of ramping will be almost $1 \%$ of the generation cost, as we see in practice (Kumar et al., 2012). We set $c_{i}^{p}=40 \$ / \mathrm{MWh}$ for all $i \in \overline{\mathcal{G}}$ and consequently $c_{i}^{r}=5.2 \$ / \mathrm{MWh}^{2}$. In what follows, we refer to the sampling method as MIP and to the robust method as SOCP.

We solve the MIP formulation with 200 randomly generated scenarios, and the SOCP formulation using Gurobi 5.6 on a desktop PC with 2.10GHz x86 64-bit Intel(R) Core(TM)2 Duo CPU and 4.00 GB RAM.

\begin{tabular}{lcccccc}
\hline Variability & \multicolumn{2}{c}{ Low } & \multicolumn{2}{c}{ Medium } & \multicolumn{2}{c}{ High } \\
\hline Methodology & MIP & SOCP & MIP & SOCP & MIP & SOCP \\
\hline Optimal Cost(1000\$/day) & 344.4 & 365.3 & 347.0 & 367.6 & 353.1 & 368.8 \\
Ramping Cost(1000\$/day) & 18.3 & 4.8 & 17.2 & 4.7 & 16.2 & 2.9 \\
Total Demand(MWh/day) & 6239 & 6239 & 6260 & 6260 & 6260 & 6260 \\
Renewable Dispatch(MWh/day) & 531 & 150 & 525 & 138 & 463 & 90 \\
Conventional Capacity(MW) & 12000 & 12000 & 12000 & 12000 & 12000 & 12000 \\
Renewable Capacity(MW) & 2160 & 2160 & 2160 & 2160 & 2160 & 2160 \\
CPU Time(sec.) & 28.0 & 0.5 & 26.3 & 0.6 & 24.2 & 0.5 \\
\hline
\end{tabular}

Table 1: 14-bus system with 10\% ramp capability and 95\% reliability 


\begin{tabular}{lcccccc}
\hline Variability & \multicolumn{2}{c}{ Low } & \multicolumn{2}{c}{ Medium } & \multicolumn{2}{c}{ High } \\
\hline Methodology & MIP & SOCP & MIP & SOCP & MIP & SOCP \\
\hline Optimal Cost(1000\$/day) & 345.1 & 366.7 & 345.8 & 367.3 & 353.5 & 369.1 \\
Ramping Cost(1000\$/day) & 16.7 & 5.3 & 16.8 & 4.6 & 16.1 & 2.7 \\
Total Demand(MWh/day) & 6250 & 6252 & 6262 & 6262 & 6260 & 6260 \\
Renewable Dispatch(MWh/day) & 518 & 152 & 531 & 142 & 459 & 83 \\
Conventional Capacity(MW) & 12000 & 12000 & 12000 & 12000 & 12000 & 12000 \\
Renewable Capacity(MW) & 2160 & 2160 & 2160 & 2160 & 2160 & 2160 \\
CPU Time(sec.) & 30.7 & 1.5 & 26.1 & 0.6 & 24.8 & 0.5 \\
\hline
\end{tabular}

Table 2: 14-bus system with 10\% ramp capability and 98\% reliability

\begin{tabular}{lcccccc}
\hline Variability & \multicolumn{2}{c}{ Low } & \multicolumn{2}{c}{ Medium } & \multicolumn{2}{c}{ High } \\
\hline Methodology & MIP & SOCP & MIP & SOCP & MIP & SOCP \\
\hline Optimal Cost(1000\$/day) & 343.3 & 366.7 & 343.2 & 367.0 & 352.8 & 368.4 \\
Ramping Cost(1000\$/day) & 28.1 & 5.0 & 28.0 & 4.7 & 17.6 & 3.1 \\
Total Demand(MWh/day) & 6248 & 6248 & 6261 & 6261 & 6264 & 6264 \\
Renewable Dispatch(MWh/day) & 674 & 146 & 697 & 145 & 485 & 103 \\
Conventional Capacity(MW) & 12000 & 12000 & 12000 & 12000 & 12000 & 12000 \\
Renewable Capacity(MW) & 2160 & 2160 & 2160 & 2160 & 2160 & 2160 \\
CPU Time(sec.) & 27.9 & 0.7 & 26.4 & 0.9 & 31.0 & 0.5 \\
\hline
\end{tabular}

Table 3: 14-bus system with $30 \%$ ramp capability and 95\% reliability

These numerical results indicate that the SOCP methodology and formulation provides more conservative solutions than the MIP one, both in total ramping and total wind power penetration. Consider the 14-bus problem. We start with the wind dispatches, as these drive many of the other results. The wind dispatch for the low wind variability in the MIP solution fluctuates in a range between $15 \mathrm{MWh}$ and $35 \mathrm{MWh}$ over the horizon (Figure 3, third panel). This contrasts with the wind power dispatches in the SOCP solution. For the low wind variability, the wind dispatch moves in a range between $5 \mathrm{MWh}$ and $10 \mathrm{MWh}$, around a third of the dispatches observed in the MIP (Figure 3). Generally, the amount of wind spilled increases for both the MIP and the SOCP when the variability of wind increases. However, the rate of change of wind spilling differs between formulations. Moving from left to right in Tables 1-4 increases the variability of the wind resource. This shows that the SOCP formulation is more conservative than the MIP, spilling larger amounts

\begin{tabular}{lcccccc}
\hline Variability & \multicolumn{2}{c}{ Low } & \multicolumn{2}{c}{ Medium } & \multicolumn{2}{c}{ High } \\
\hline Methodology & MIP & SOCP & MIP & SOCP & MIP & SOCP \\
\hline Optimal Cost $(\$ 1000 /$ day) & 345.8 & 367.7 & 344.6 & 367.5 & 352.6 & 368.8 \\
Ramping Cost(\$1000/day) & 26.4 & 5.0 & 27.4 & 4.7 & 18.8 & 3.0 \\
Total Demand(MWh/day) & 6266 & 6266 & 6258 & 6258 & 6260 & 6260 \\
Renewable Dispatch(MWh/day) & 643 & 148 & 673 & 139 & 503 & 92 \\
Conventional Capacity(MW) & 12000 & 12000 & 12000 & 12000 & 12000 & 12000 \\
Renewable Capacity(MW) & 2160 & 2160 & 2160 & 2160 & 2160 & 2160 \\
CPU Time(sec.) & 26.9 & 0.6 & 27.6 & 0.5 & 25.6 & 0.5 \\
\hline
\end{tabular}

Table 4: 14-bus system with $30 \%$ ramp capability and $98 \%$ reliability 


\begin{tabular}{lcccccc}
\hline Variability & \multicolumn{2}{c}{ Low } & \multicolumn{2}{c}{ Medium } & \multicolumn{2}{c}{ High } \\
\hline Methodology & MIP & SOCP & MIP & SOCP & MIP & SOCP \\
\hline Optimal Cost(\$1000/day) & 279.3 & 294.0 & 281.9 & 294.7 & 285.8 & 294.2 \\
Ramping Cost(\$1000/day) & 2.9 & 6.3 & 4.3 & 5.9 & 5.2 & 3.9 \\
Total Demand(MWh/day) & 5120 & 5120 & 5103 & 5103 & 5090 & 5090 \\
Renewable Dispatch(MWh/day) & 226 & 155 & 233 & 124 & 223 & 71 \\
Conventional Capacity(MW) & 6264 & 6264 & 6264 & 6264 & 6264 & 6264 \\
Renewable Capacity(MW) & 1128 & 1128 & 1128 & 1128 & 1128 & 1128 \\
CPU Time(sec.) & 347.7 & 5.6 & 143.7 & 2.0 & 75.6 & 2.0 \\
\hline
\end{tabular}

Table 5: 30-bus system with $10 \%$ ramp capability and 95\% reliability

\begin{tabular}{lcccccc}
\hline Variability & \multicolumn{2}{c}{ Low } & \multicolumn{2}{c}{ Medium } & \multicolumn{2}{c}{ High } \\
\hline Methodology & MIP & SOCP & MIP & SOCP & MIP & SOCP \\
\hline Optimal Cost(1000\$/day) & 279.3 & 294.0 & 280.6 & 293.6 & 282.0 & 291.2 \\
Ramping Cost(1000\$/day) & 2.9 & 6.3 & 3.1 & 5.2 & 4.5 & 3.5 \\
Total Demand(MWh/day) & 5120 & 5120 & 5122 & 5122 & 5080 & 5080 \\
Renewable Dispatch(MWh/day) & 226 & 155 & 237 & 150 & 237 & 84 \\
Conventional Capacity(MW) & 6260 & 6260 & 6260 & 6260 & 6260 & 6260 \\
Renewable Capacity(MW) & 1128 & 1128 & 1128 & 1128 & 1128 & 1128 \\
CPU Time(sec.) & 193.1 & 3.4 & 131.8 & 2.4 & 206.1 & 11.1 \\
\hline
\end{tabular}

Table 6: 30-bus system with 10\% ramp capability and $98 \%$ reliability

of wind as the variability of wind increases.

In terms of the financial costs of the models, the amount of wind absorbed by the system partly explains the main differences between the MIP and the SOCP formulation. Larger amounts of wind dispatched results in larger ramping costs for the MIP model, ranging between $\$ 16.7$ and $\$ 28.1$ Thousand/day for the low wind variability 14-bus system case in Tables 1-4. On the other hand, for the SOCP formulation the ramping costs are between $\$ 4.8$ and $\$ 5.3$ Thousand/day in three of the cases shown. There is a trade-off in the ramping cost incurred versus the amount of wind dispatched as the variability increases, with higher ramping costs offset by more wind spilling.

The overall conservativeness of the SOCP solution and consequent spilling of wind also leads to consistently and marginally higher total operational costs compared to the MIP. Referring to

\begin{tabular}{lcccccc}
\hline Variability & \multicolumn{2}{c}{ Low } & \multicolumn{2}{c}{ Medium } & \multicolumn{2}{c}{ High } \\
\hline Methodology & MIP & SOCP & MIP & SOCP & MIP & SOCP \\
\hline Optimal Cost(1000\$/day) & 250.2 & 293.8 & 257.0 & 293.4 & 275.2 & 292.8 \\
Ramping Cost(1000\$/day) & 15.6 & 7.7 & 15.5 & 5.4 & 13.8 & 3.8 \\
Total Demand(MWh/day) & 5099 & 5099 & 5108 & 5108 & 5097 & 5097 \\
Renewable Dispatch(MWh/day) & 699 & 154 & 705 & 140 & 587 & 92 \\
Conventional Capacity(MW) & 6264 & 6264 & 6264 & 6264 & 6264 & 6264 \\
Renewable Capacity(MW) & 1128 & 1128 & 1128 & 1128 & 1128 & 1128 \\
CPU Time(sec.) & 186.5 & 2.0 & 162.6 & 2.8 & 128.7 & 2.0 \\
\hline
\end{tabular}

Table 7: 30-bus system with $30 \%$ ramp capability and $95 \%$ reliability 


\begin{tabular}{lcccccc}
\hline Variability & \multicolumn{2}{c}{ Low } & \multicolumn{2}{c}{ Medium } & \multicolumn{2}{c}{ High } \\
\hline Methodology & MIP & SOCP & MIP & SOCP & MIP & SOCP \\
\hline Optimal Cost(1000\$/day) & 252.6 & 296.0 & 254.4 & 290.7 & 280.8 & 296.3 \\
Ramping Cost(1000\$/day) & 15.3 & 6.8 & 15.4 & 5.4 & 14.5 & 3.9 \\
Total Demand(MWh/day) & 5119 & 5119 & 5087 & 5087 & 5113 & 5113 \\
Renewable Dispatch(MWh/day) & 695 & 148 & 711 & 138 & 575 & 72 \\
Conventional Capacity(MW) & 6264 & 6264 & 6264 & 6264 & 6264 & 6264 \\
Renewable Capacity(MW) & 1128 & 1128 & 1128 & 1128 & 1128 & 1128 \\
CPU Time(sec.) & 193.6 & 3.1 & 149.7 & 2.0 & 128.6 & 2.5 \\
\hline
\end{tabular}

Table 8: 30-bus system with 30\% ramp capability and $98 \%$ reliability

\begin{tabular}{lcccccc}
\hline Variability & \multicolumn{2}{c}{ Low } & \multicolumn{2}{c}{ Medium } & \multicolumn{2}{c}{ High } \\
\hline Methodology & MIP & SOCP & MIP & SOCP & MIP & SOCP \\
\hline Optimal Cost(1000\$/day) & - & 690.1 & - & 689.8 & - & 689.2 \\
Ramping Cost(1000\$/day) & - & 1.0 & - & 1.2 & - & 0.9 \\
Total Demand(MWh/day) & - & 35686 & - & 35677 & - & 35654 \\
Renewable Dispatch(MWh/day) & - & 110 & - & 115 & - & 94 \\
Conventional Capacity(MW) & - & 128424 & - & 128424 & - & 128424 \\
Renewable Capacity(MW) & - & 24000 & - & 24000 & - & 24000 \\
CPU Time (sec.) & - & 44.1 & - & 55.2 & - & 46.4 \\
\hline
\end{tabular}

Table 9: 118-bus system with $10 \%$ ramp capability and $95 \%$ reliability

the 14-bus system in Tables 1-4, and focusing on the low variability cases, the MIP costs oscillate between $\$ 343.3$ and $\$ 345.8$ Thousand/day, versus costs of the SOCP cases ranging from $\$ 365.3$ to $\$ 367.7$ Thousand/day. Comparing Tables 1-2 to Tables 3-4 allows to understand the effect of increasing the ramp capability of the system. The increase in ramping capability does affect the overall cost observed as the wind variability increases. However, as the reliability level increases, this effect is muted.

The last row of Tables 1-14 provides evidence of the one of the main benefits of our approach: there are significant gains in the solution time obtained when using the SOCP method instead of the MIP, around 50 times faster. It turns out that the MIP formulation of the 118-bus system cannot be solved in reasonable time (less than 10000 seconds) even if we reduce the number of scenarios. Thus, we report only the solution of the SOCP formulation for the 118-bus system. We

\begin{tabular}{lcccccc}
\hline Variability & \multicolumn{2}{c}{ Low } & \multicolumn{2}{c}{ Medium } & \multicolumn{2}{c}{ High } \\
\hline Methodology & MIP & SOCP & MIP & SOCP & MIP & SOCP \\
\hline Optimal Cost(1000\$/day) & - & 688.7 & - & 688.6 & - & 688.3 \\
Ramping Cost(1000\$/day) & - & 1.0 & - & 1.2 & - & 1.2 \\
Total Demand(MWh/day) & - & 35638 & - & 35635 & - & 35621 \\
Renewable Dispatch(MWh/day) & - & 110 & - & 118 & - & 92 \\
Conventional Capacity(MW) & - & 128424 & - & 128424 & - & 128424 \\
Renewable Capacity(MW) & - & 24000 & - & 24000 & - & 24000 \\
CPU Time(sec.) & - & 49.4 & - & 46.6 & - & 44.8 \\
\hline
\end{tabular}

Table 10: 118-bus system with $10 \%$ ramp capability and $99.97 \%$ reliability 


\begin{tabular}{lcccccc}
\hline Variability & \multicolumn{2}{c}{ Low } & \multicolumn{2}{c}{ Medium } & \multicolumn{2}{c}{ High } \\
\hline Methodology & MIP & SOCP & MIP & SOCP & MIP & SOCP \\
\hline Optimal Cost(1000\$/day) & - & 690.4 & - & 689.5 & - & 690.1 \\
Ramping Cost(1000\$/day) & - & 1.1 & - & 1.2 & - & 0.9 \\
Total Demand(MWh/day) & - & 35691 & - & 35665 & - & 35681 \\
Renewable Dispatch(MWh/day) & - & 110 & - & 117 & - & 85 \\
Conventional Capacity(MW) & - & 128424 & - & 128424 & - & 128424 \\
Renewable Capacity(MW) & - & 24000 & - & 24000 & - & 24000 \\
CPU Time(sec.) & - & 33.8 & - & 35.8 & - & 37.9 \\
\hline
\end{tabular}

Table 11: 118-bus system with $30 \%$ ramp capability and $95 \%$ reliability

\begin{tabular}{lcccccc}
\hline Variability & \multicolumn{2}{c}{ Low } & \multicolumn{2}{c}{ Medium } & \multicolumn{2}{c}{ High } \\
\hline Methodology & MIP & SOCP & MIP & SOCP & MIP & SOCP \\
\hline Optimal Cost(1000\$/day) & - & 689.8 & - & 690.1 & - & 690.0 \\
Ramping Cost(1000\$/day) & - & 1.1 & - & 1.1 & - & 1.1 \\
Total Demand(MWh/day) & - & 35673 & - & 35695 & - & 35679 \\
Renewable Dispatch(MWh/day) & - & 108 & - & 119 & - & 86 \\
Conventional Capacity(MW) & - & 128424 & - & 128424 & - & 128424 \\
Renewable Capacity(MW) & - & 24000 & - & 24000 & - & 24000 \\
CPU Time(sec.) & - & 49.9 & - & 49.7 & - & 47.2 \\
\hline
\end{tabular}

Table 12: 118-bus system with $30 \%$ ramp capability and $99.97 \%$ reliability

provide robustness checks in Tables 5-14, using the IEEE 30 bus and 118 bus systems.

In practice, the typical reliability value used by NERC is one day in ten years, which accrues to a probability of approximately $1-\gamma=0.9997$. Tables 10 and 12 provide the result for this reliability level in the 118-bus system. Additionally, different conventional power plants are likely to be quite heterogeneous in terms of their ramping costs. To test this instance, we run the experiment with $c_{i}^{r}=0.13 c_{i}^{p}+c_{n}$ where $c_{n}$ is a random normal variable with mean zero and standard deviation five, i.e., $c \sim \mathcal{N}(0,5)$. Tables $13-14$ show analogous results to the case with uniform ramping for the case of heterogeneous ramping costs, at the $98 \%$ reliability level.

The overall trends already described for the 14-bus system are similar for the additional test networks. However, as the size of the system increases, the MIP methodology and formulation becomes ineffective in terms of computational performance. In addition, the results show that as

\begin{tabular}{lcccccc}
\hline Variability & \multicolumn{2}{c}{ Low } & \multicolumn{2}{c}{ Medium } & \multicolumn{2}{c}{ High } \\
\hline Methodology & MIP & SOCP & MIP & SOCP & MIP & SOCP \\
\hline Optimal Cost(1000\$/day) & - & 691.9 & - & 691.1 & - & 691.4 \\
Ramping Cost(1000\$/day) & - & 1.1 & - & 0.8 & - & 1.2 \\
Total Demand(MWh/day) & - & 35694 & - & 35679 & - & 35674 \\
Renewable Dispatch(MWh/day) & - & 23 & - & 21 & - & 21 \\
Conventional Capacity(MW) & - & 128424 & - & 128424 & - & 128424 \\
Renewable Capacity(MW) & - & 24000 & - & 24000 & - & 24000 \\
CPU Time(sec.) & - & 48.4 & - & 51.6 & - & 50.1 \\
\hline
\end{tabular}

Table 13: 118-bus system with $10 \%$ ramp capability and $98 \%$ reliability with random ramping cost 


\begin{tabular}{lcccccc}
\hline Variability & \multicolumn{2}{c}{ Low } & \multicolumn{2}{c}{ Medium } & \multicolumn{2}{c}{ High } \\
\hline Methodology & MIP & SOCP & MIP & SOCP & MIP & SOCP \\
\hline Optimal Cost(1000\$/day) & - & 689.8 & - & 691.2 & - & 690.5 \\
Ramping Cost(1000\$/day) & - & 0.8 & - & 0.9 & - & 1.0 \\
Total Demand(MWh/day) & - & 35633 & - & 35678 & - & 35647 \\
Renewable Dispatch(MWh/day) & - & 20 & - & 22 & - & 19 \\
Conventional Capacity(MW) & - & 128424 & - & 128424 & - & 128424 \\
Renewable Capacity(MW) & - & 24000 & - & 24000 & - & 24000 \\
CPU Time(sec.) & - & 42.4 & - & 46.7 & - & 45.2 \\
\hline
\end{tabular}

Table 14: 118-bus system with $30 \%$ ramp capability and $98 \%$ reliability with random ramping cost

the wind variability increases, the SOCP and MIP methodologies dispatch slightly less wind power to reduce the cost and damaging effects of ramping.
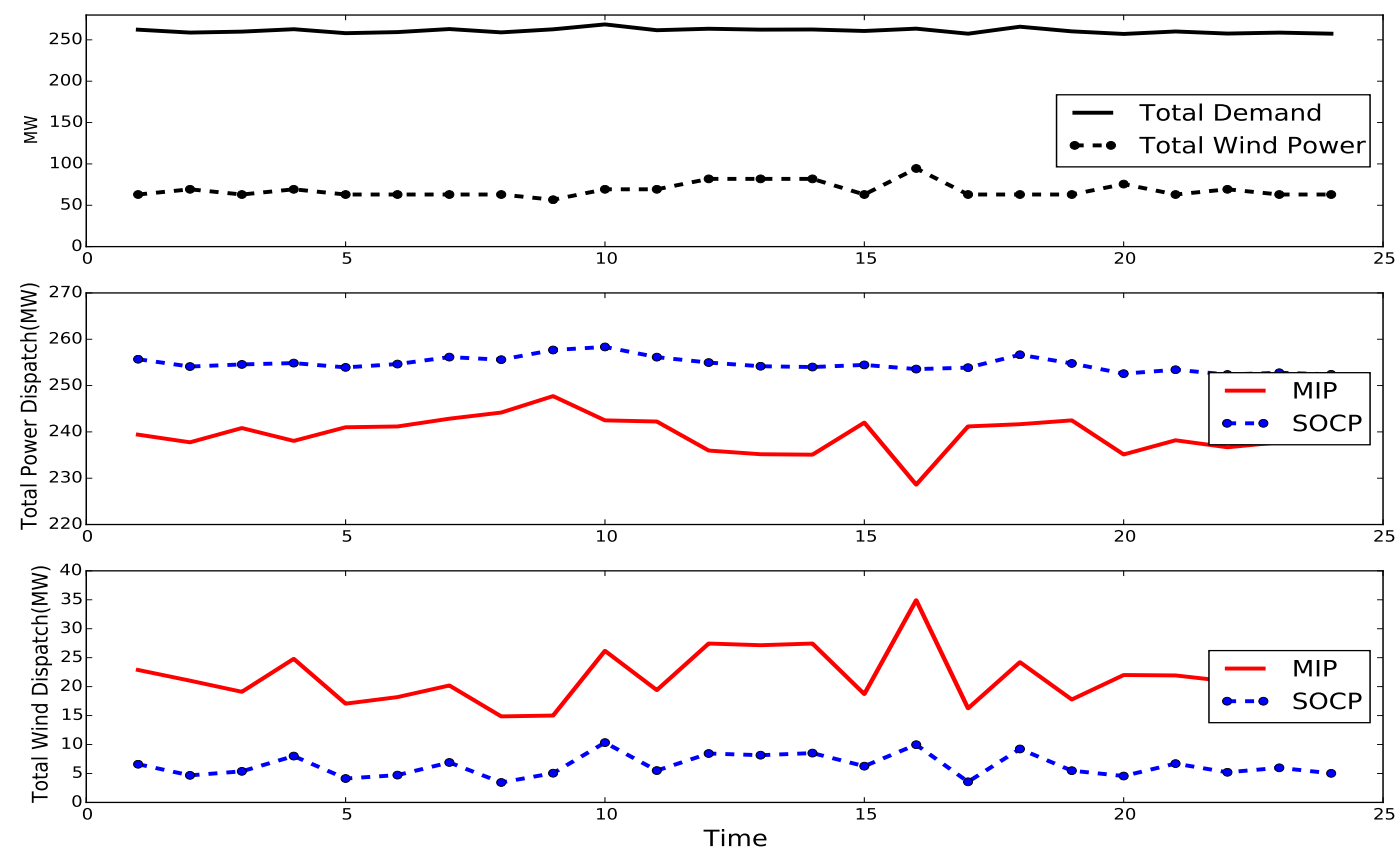

Figure 3: 14-bus system under low wind variability with 10\% ramp capability and $98 \%$ reliability

Figures 3-8 show the changes in power dispatch of conventional generators and wind generators for $98 \%$ reliability and $10 \%$ ramp capability for the 14 -bus and 30-bus systems. Consider the 14-bus system. The first plot in Figures 3-5 shows the demand profile considered and the variability of wind for the three variability levels considered. The demand profile modeled is relatively constant to isolate the effects of demand variability; and the wind variability output progressively increases from Figure 3 to Figure 5. The second and third plots compare the hourly dispatch from conventional and wind generators for the MIP and SOCP methods. The hourly dispatch trends are consistent with the results observed in daily totals, with the MIP dispatching more wind and therefore less conventional generation, while the SOCP generally spills more wind at all hours. It is remarkable how the variability of the absorbed wind in the system increases the ramping of the 

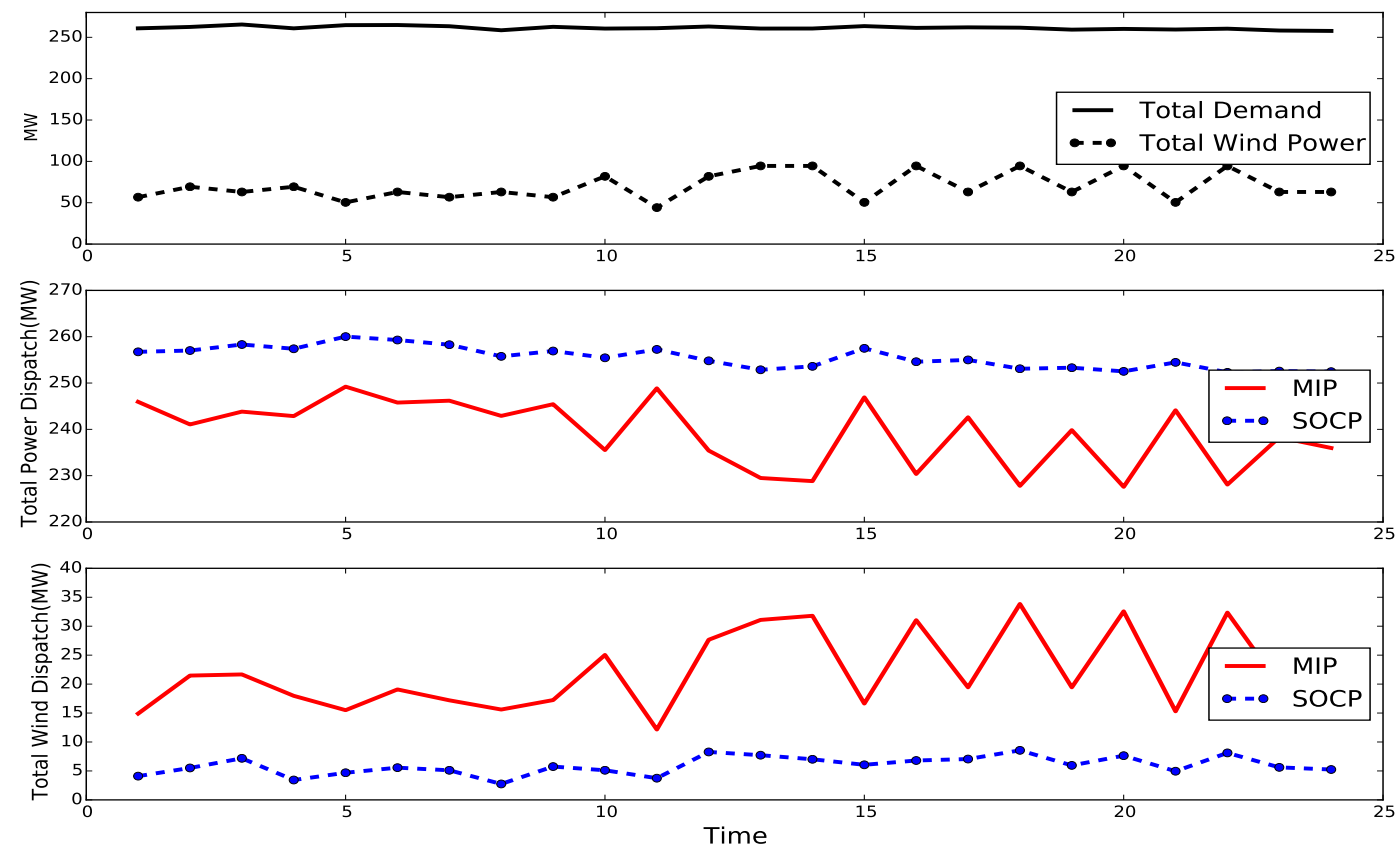

Figure 4: 14-bus system under medium wind variability with 10\% ramp capability and $98 \%$ reliability

conventional generation in the MIP, in contrast with the generally regular output of both wind and conventional generation in the SOCP.

\section{Conclusion}

In this work, we study ramping costs and constraints along with their effects on generation and dispatch policies in an electricity network with uncertain renewable energy sources. Specifically, we consider an economic dispatch problem in which the objective is to minimize the operational costs of the system while maintaining a high reliability level. First, we show that ramping can have a significant effect on the renewable dispatch policy. Then, we propose a robust optimization model to address a general ramp-constrained economic dispatch problem taking into account the inherent uncertainty of the renewable energy sources available. Furthermore, we show that for practical instances of the economic dispatch problem, the dispatch policy can be found by solving a convex optimization problem. To illustrate the practical value of our approach, we compare the performance of the dispatch policies obtained in this form, with dispatch policies obtained by modeling the uncertainty of the RES output using a sample of scenarios. The numerical experiments presented here show that the robust approach allows for the solution of much larger networks than the typical sampling solution approach in terms of computational effort. Not surprisingly, the results indicate that the robust approach provides more conservative dispatch policies than the sampling solution approach. Interestingly, this robustness leads to dispatch solutions that require less ramping with little trade-off in terms of long-term expected costs of the network.

For future research, it is necessary to obtain and estimate more accurate ramping costs for different conventional power plants. 

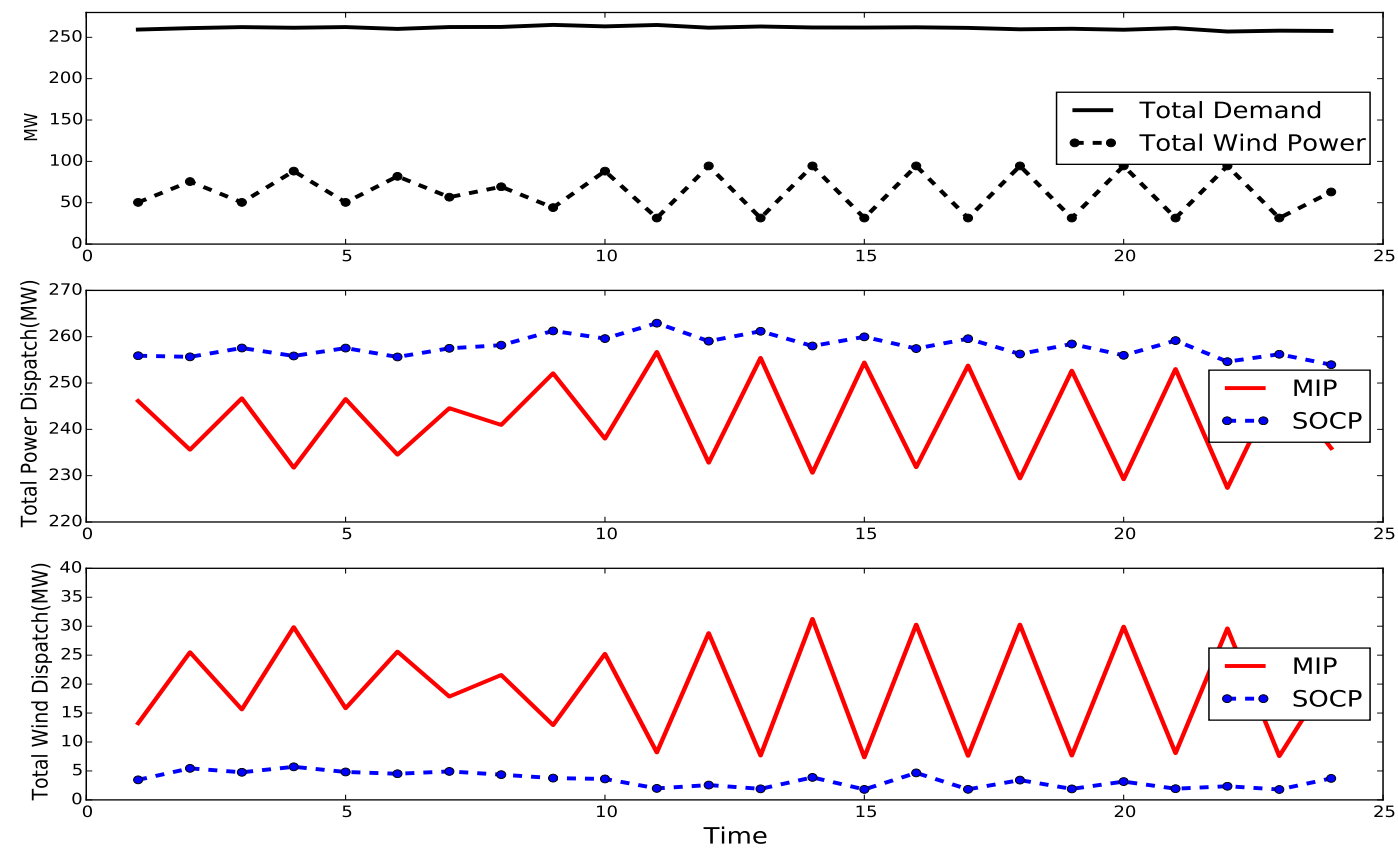

Figure 5: 14-bus system under high wind variability with 10\% ramp capability and $98 \%$ reliability

Additionally, it is of interest to consider other renewable sources such as wave and solar. For those cases where less accurate distributional information about the RES uncertainty is available, one can make use of the multivariate Chebychev-Cantelly inequality (Lanckriet et al., 2003, Lemma 1), to obtain an SOCP model for the stochastic ED problem similar to the one presented here in the case of wind. However, because in this instance the chance constraints in the model would be satisfied against the worst possible realization of the resource in consideration, the main tradeoff is that this method is more conservative than our approach and can lead to unnecessarily higher costs.

\section{Acknowledgements}

We thank the editor and two anonymous reviewers for their constructive suggestions. This research was supported by the the Lehigh Faculty Innovation Grant, the National Science Foundation through the CyberSEES grant \#1442858, and the US Department of Energy through the Consortium for Electric Reliability Technology Solutions (CERTS). The authors are responsible for all conclusions presented in the article, and the views expressed have not been endorsed by the sponsoring agencies.

\section{References}

S. Ahmed and A. Shapiro. Solving chance-constrained stochastic programs via sampling and integer programming. Tutorials in Operations Research: State-of-the-art Decision-making Tools in the Information-intensive Age, 2008. 

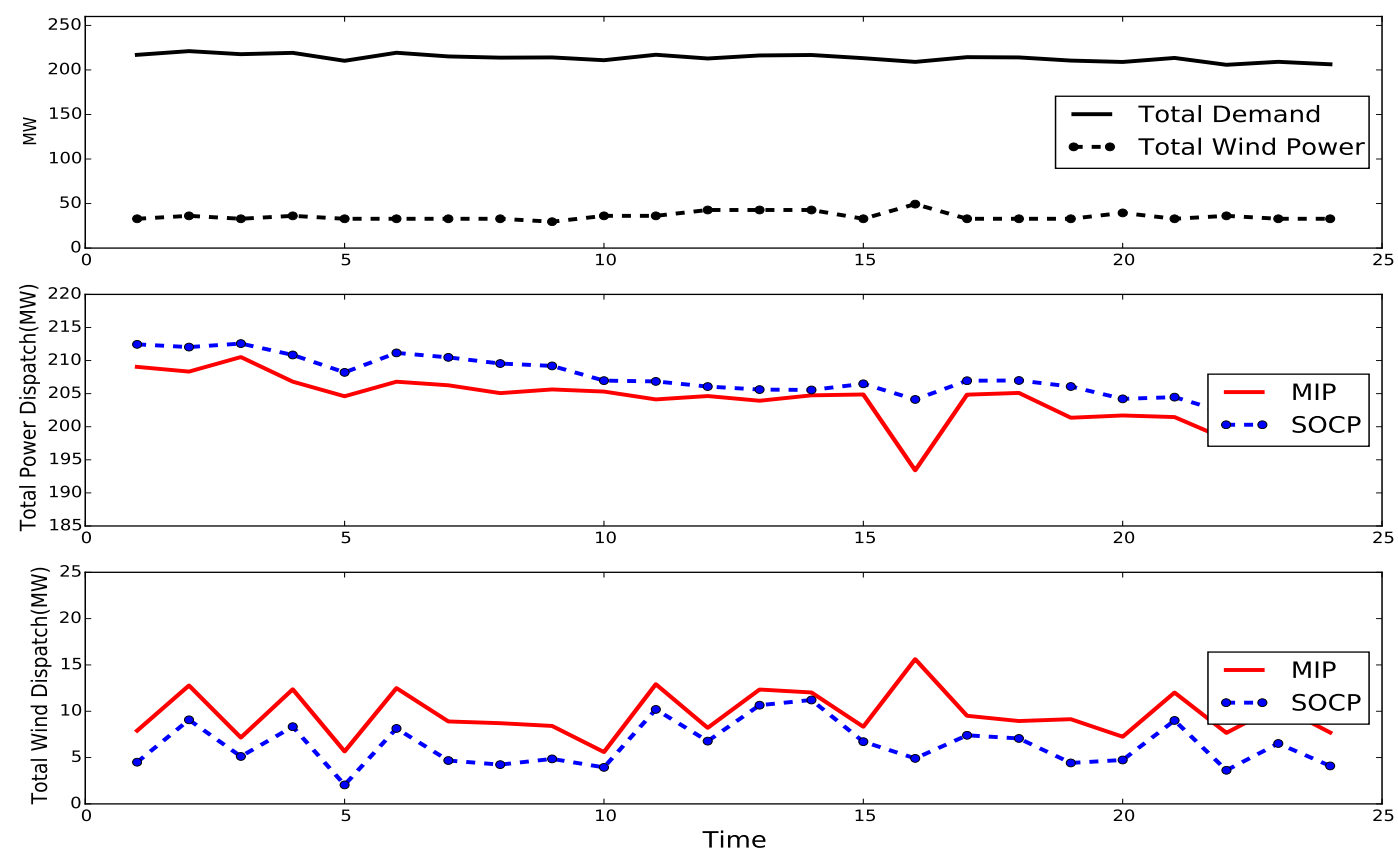

Figure 6: 30 -bus system under low wind variability with $10 \%$ ramp capability and $98 \%$ reliability

P. Attaviriyanupap, H. Kita, E. Tanaka, and J. Hasegawa. A hybrid ep and sqp for dynamic economic dispatch with nonsmooth fuel cost function. Power Systems, IEEE Transactions on, 17(2):411-416, May 2002. ISSN 0885-8950. doi: 10.1109/TPWRS.2002.1007911.

T. Barbounis, J. Theocharis, M. Alexiadis, and P. Dokopoulos. Long-term wind speed and power forecasting using local recurrent neural network models. Energy Conversion, IEEE Transactions on, 21(1):273-284, 2006.

A. Ben-Tal, L. El Ghaoui, and A.S. Nemirovski. Robust Optimization. Princeton Series in Applied Mathematics. Princeton University Press, October 2009.

D. Bienstock, M. Chertkov, and S. Harnett. Chance constrained optimal power flow: Risk-aware network control under uncertainty. September 2012. URL http://arxiv.org/pdf/1209.5779. pdf.

J.R. Birge and F. Louveaux. Introduction to Stochastic Programming. Springer Series in Operations Research Series. Springer London, Limited, 1997. ISBN 9780387982175. URL http://books . google.com/books?id=cfrMw9crazsC.

P. Bonami and M. A. Lejeune. An exact solution approach for portfolio optimization problems under stochastic and integer constraints. Operations research, 57(3):650-670, 2009.

Stephen Boyd and Lieven Vandenberghe. Convex Optimization. Cambridge University Press, 2009. URL http://www.stanford.edu/ boyd/cvxbook/bv_cvxbook.pdf.

M. Cain, R. O’Neill, and A. Castillo. History of optimal power flow and formulations. 2012a. 

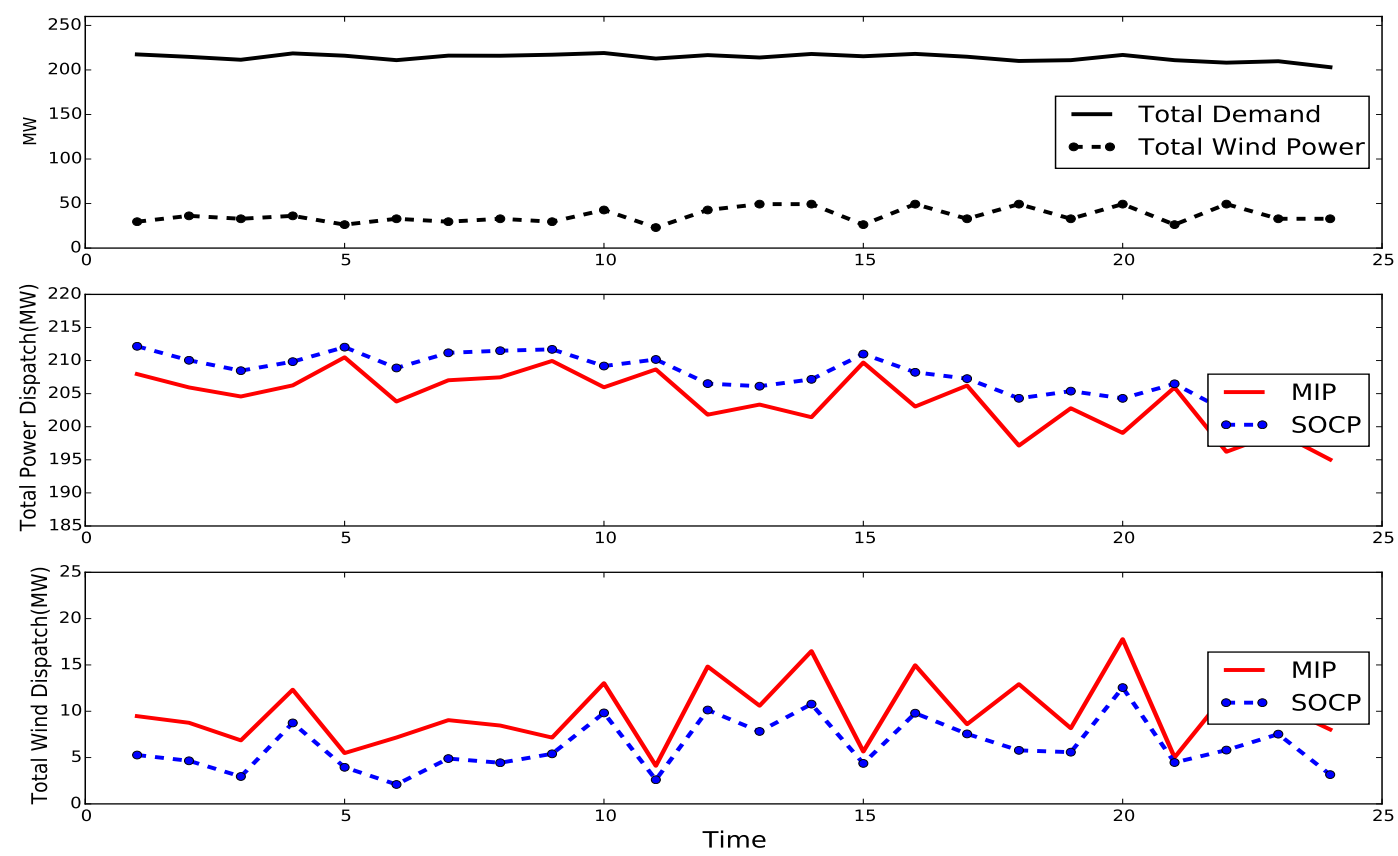

Figure 7: 30-bus system under medium wind variability with 10\% ramp capability and $98 \%$ reliability

Mary B. Cain, Richard P. O’Neill, and Anya Castillo. History of optimal power flow and formulations. Technical report, FERC, 2012b. URL http://www.ferc.gov/industries/electric/ indus-act/market-planning/opf-papers/acopf-1-history-formulation-testing.pdf.

J. Carpentier. Contribution a l'etude du dispatching economique. Bulletin de la Societe Francaise des Electriciens, 3:431-447, 1962.

A. Charnes and W. Cooper. Deterministic equivalents for optimizing and satisfying under chance constraints. Operations Research, 11(1):pp. 18-39, 1963.

A. Dorvlo. Estimating wind speed distribution. Energy Conversion and Management, 43(17): 2311-2318, 2002.

E. Ela and M. O'Malley. Studying the variability and uncertainty impacts of variable generation at multiple timescales. Power Systems, IEEE Transactions on, 27(3):1324-1333, Aug 2012. ISSN 0885-8950. doi: 10.1109/TPWRS.2012.2185816.

T. P. Filomena and M. A. Lejeune. Warm-start heuristic for stochastic portfolio optimization with fixed and proportional transaction costs. Journal of Optimization Theory and Applications, 161 (1):308-329, 2014.

A. Garcia, J. Torres, E. Prieto, and A. De Francisco. Fitting wind speed distributions: a case study. Solar energy, 62(2):139-144, 1998.

J. Hennessey and P. Joseph. Some aspects of wind power statistics. Journal of applied meteorology, 16(2):119-128, 1977. 

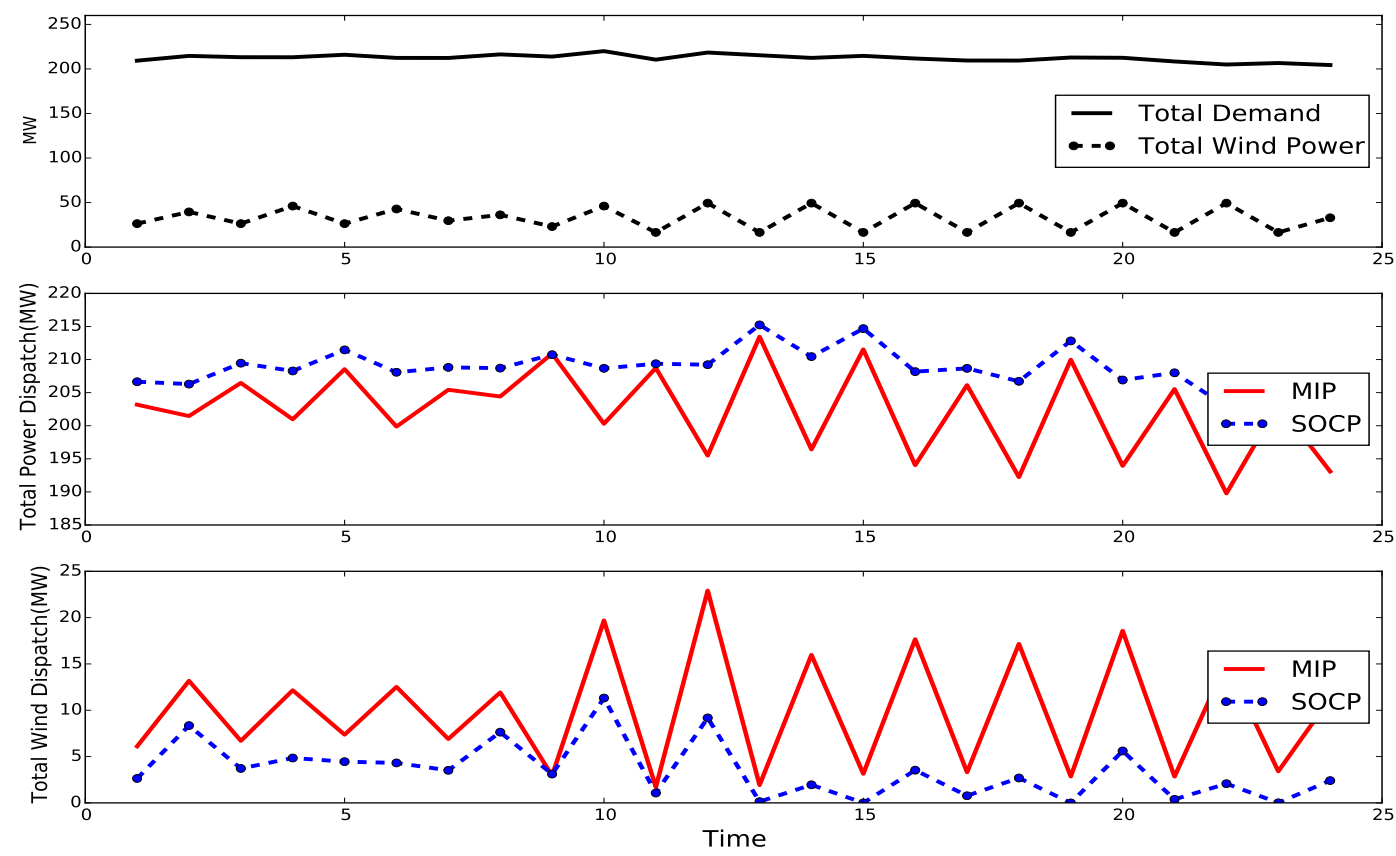

Figure 8: 30-bus system under high wind variability with 10\% ramp capability and $98 \%$ reliability

J. Hetzer, D. Yu, and K. Bhattarai. An economic dispatch model incorporating wind power. IEEE Transactions on Energy Conversion, 23(2):603-611, June 2008. ISSN 0885-8969. doi: 10.1109/TEC.2007.914171.

M. Huneault and F.D. Galiana. A survey of the optimal power flow literature. Power Systems, IEEE Transactions on, 6(2):762-770, May 1991. ISSN 0885-8950. doi: 10.1109/59.76723.

G. Irisarri, L. M. Kimball, K. A. Clements, A. Bagchi, and P. W. Davis. Economic dispatch with network and ramping constraints via interior point methods. IEEE Transactions on Power Systems, 13(1):236-242, 1998.

G. Jordan and S. Venkataraman. Analysis of Cycling Costs in Western Wind and Solar Integration Study. GE Energy prepared for NREL, 2012.

C. Justus, W. Hargraves, A. Mikhail, and D. Graber. Methods for estimating wind speed frequency distributions. Journal of applied meteorology, 17(3):350-353, 1978.

T. Kumano. A functional optimization based dynamic economic load dispatch considering ramping rate of thermal units output. In Power Systems Conference and Exposition (PSCE), pages 1-8, March 2011. doi: 10.1109/PSCE.2011.5772540.

N. Kumar, P. Besuner, S. Lefton, D. Agan, and D. Hilleman. Power Plant Cycling Costs. Intertek APTECH prepared for NREL, 2012.

A. Lamadrid, T. Mount, W. Jeon, and H. Lu. Is deferrable demand an effective alternative to upgrading transmission capacity? Journal of Energy Engineering, page B4014005, 2015/02/05 
2014. doi: 10.1061/(ASCE)EY.1943-7897.0000182. URL http://dx.doi.org/10.1061/(ASCE) EY.1943-7897.0000182.

Alberto J. Lamadrid and Tim Mount. Ancillary services in systems with high penetrations of renewable energy sources, the case of ramping. Energy Economics, 34(6):1959 - 1971, 2012. ISSN 0140-9883. doi: 10.1016/j.eneco.2012.08.011. URL http://www.sciencedirect.com/ science/article/pii/S0140988312001818.

Gert R.G. Lanckriet, Laurent El Ghaoui, Chiranjib Bhattacharyya, and Michael I. Jordan. A robust minimax approach to classification. J. Mach. Learn. Res., 3:555-582, 2003. ISSN 15337928.

S. Lefton. Cost analysis and cost-based power plant asset management-thermal power plant cycling costs. In APTECH, 2011.

S. Lefton and D. Hilleman. Make your plant ready for cycling operations. Power, 2011.

S. Lefton, P. Grimsrud, P. Besuner, D. Agan, and J. Grover. Analysis of cycling impacts on combined cycle. In ASME Power, 2008.

D. Lew, G. Brinkman, N. Kumnar, P. Besuner, D. Agan, and S. A. Lefton. Impacts of Wind and Solar on Fossil-Fueled Generators. IEEE Power and Energy Society General Meeting, 2012.

D. Lew, G. Brinkman, E. Ibanez, A. Florita, M. Heaney, B. M. Hodge, M. Hummon, G. Stark, J. King, S. Lefton, N. Kumar, D. Agan, G. Jordan, and S. Venkataraman. The Western Wind and Solar Integration Study Phase 2. NREL Technical Report, 2013.

H. Louie and J. Sloughter. Probabilistic modeling and statistical characteristics of aggregate wind power. In Large Scale Renewable Power Generation, pages 19-51. Springer, 2014.

J. Luedtke. An integer programming and decomposition approach to general chance-constrained mathematical programs. In Integer Programming and Combinatorial Optimization, pages 271284. Springer, 2010.

J. Luedtke, S. Ahmed, and G. Nemhauser. An integer programming approach for linear programs with probabilistic constraints. In Integer Programming and Combinatorial Optimization, pages 410-423. Springer, 2007.

S. Majumdar, D. Chattopadhyay, and J. Parikh. Interruptible load management using optimal power flow analysis. IEEE Transactions on Power Systems, 11(2):715-720, May 1996. ISSN 0885-8950. doi: 10.1109/59.496144.

G. Masters. Renewable and efficient electric power systems. John Wiley \& Sons, 2013.

P. Meibom, R. Barth, B. Hasche, H. Brand, C. Weber, and M. O'Malley. Stochastic optimization model to study the operational impacts of high wind penetrations in ireland. Power Systems, IEEE Transactions on, PP(99):1 -12, 2010. ISSN 0885-8950. doi: 10.1109/TPWRS.2010. 2070848. 
M. Moarefdoost and L. Snyder. Generation and Storage Dispatch in Electricity Networks with Generator Disruptions. Naval Research Logistics, 62:493-511, 2015.

E. Morgan, M. Lackner, R. Vogel, and L. Baise. Probability distributions for offshore wind speeds. Energy Conversion and Management, 52(1):15-26, 2011.

N. Navid and G. Rosenwald. Market Solutions for Managing Ramp Flexibility With High Penetration of Renewable Resource. IEEE Tansactions on Sustainable Energy, 3(4):784-790, 2012.

Per Norgaard and Hannele Hottlinen. A multi-turbine power curve approach. In Nordic Wind Power Conference, pages 1-5. http://www.ieawind.org/index.html, 2004.

A. Papavasiliou, S.S. Oren, and R.P. O’Neill. Reserve requirements for wind power integration: A scenario-based stochastic programming framework. Power Systems, IEEE Transactions on, 26 (4):2197 -2206, nov. 2011. ISSN 0885-8950. doi: 10.1109/TPWRS.2011.2121095.

M. Schuerger, H. Johal, L. Roose, M. Matsuura, and R. Piwko. Catching some rays: Variable generation integration on the island of oahu. Power and Energy Magazine, IEEE, 11(6):33-44, 2013. ISSN 1540-7977. doi: 10.1109/MPE.2013.2277998.

J. V. Seguro and T. W. Lambert. Modern estimation of the parameters of the weibull wind speed distribution for wind energy analysis. Journal of Wind Engineering and Industrial Aerodynamics, $85(1): 75-84,2000$.

G. B. Sherestha, K. Song, and L. Goel. Strategic self-dispatch considering ramping costs in deregulated power markets. IEEE Transaction on Power Systems, 19(3), 2004.

A. Shibli, J. Gostling, F. Starr, V. Viswanathan, and D. Gray. Damage to Power Plants Due to Cycling. Electric Power Research Institute, www.epri.com, 2001.

M. Tanaka. Real-time pricing with ramping costs: A new approach to managing a steep change in electricity demand. Energy Policy, 34:3634-3643, 2006.

N. Troy, D. Flynn, M. Milligan, and M. O'Malley. Unit commitment with dynamic cycling costs. Power Systems, IEEE Transactions on, PP(99):1, 2012. ISSN 0885-8950. doi: 10.1109/TPWRS. 2012.2192141.

S. Tuller and A. Brett. The characteristics of wind velocity that favor the fitting of a weibull distribution in wind speed analysis. Journal of Climate and Applied Meteorology, 23(1):124-134, 1984.

R. Viswanathan. Damage Mechanisms and Life Assessment of High-Temperature Components. ASM International, 1989.

C. Wang and S. M. Shahidehpour. Optimal generation scheduling with ramping costs. Power Systems, IEEE Transactions on, 10(1):60-67, 1995.

Y. Zuwei and A. Tuzuner. Fractional weibull wind speed modeling for wind power production estimation. In Power Energy Society General Meeting, pages 1-7, July 2009. doi: 10.1109/PES. 2009.5275785 . 


\section{Appendix A. Scenario Based Formulation}

One approach to write the deterministic equivalent of a chance constrained optimization problem is by defining a set of binary variables corresponding to the set of possible scenarios. Let $\Pi_{j t}=\left\{\pi_{j t}^{1}, \pi_{j t}^{2}, \ldots, \pi_{j t}^{m}\right\}$ be the set of possible scenarios for $\varepsilon_{j}^{t}$ in time $t, \forall j \in \tilde{\mathcal{G}}$. We define binary variables $\bar{z}_{s}^{t}, \hat{z}_{s}^{t}$ and $\tilde{z}_{s}^{t}$ for $s=1,2, \cdots, m$ and $t=1,2, \cdots, T$ as:

$$
\bar{z}_{s}^{t}= \begin{cases}0 & \text { if }-\bar{f}_{l} \leq \sum_{i \in \overline{\mathcal{G}}} \hat{B}_{l i}^{\bar{g}}\left(\bar{p}_{i}^{t}+\frac{1}{|\mathcal{G}|} \sum_{j \in \tilde{\mathcal{G}}} x_{j}^{t} \pi_{j t}^{s}\right)+\sum_{j \in \tilde{\mathcal{G}}} \hat{B}_{l j}^{\tilde{g}} x_{j}^{t} \pi_{j t}^{s}-\sum_{i \in \mathcal{D}} \hat{B}_{l i}^{d} d_{i}^{t} \leq \bar{f}_{l}, \forall l \in \mathcal{L} \\ 1 & \text { Otherwise }\end{cases}
$$

for the probabilistic constraints (26) and (27).

$$
\tilde{z}_{s}^{t}= \begin{cases}0 & \text { if } r_{i}^{m i n} \leq \bar{r}_{i}^{t} \tau-\frac{1}{|\mathcal{G}|} \sum_{j \in \tilde{\mathcal{G}}} x_{j}^{t} \pi_{j t}^{s}+\frac{1}{|\mathcal{G}|} \sum_{j \in \tilde{\mathcal{G}}} x_{j}^{t-1} \pi_{j t-1}^{s} \leq r_{i}^{\text {max }}, \forall i \in \overline{\mathcal{G}} \\ 1 & \text { Otherwise }\end{cases}
$$

for the probabilistic constraints (30) and (31). And,

$$
\hat{z}_{s}^{t}= \begin{cases}0 & \text { if } P_{i}^{m i n} \leq \bar{p}_{i}^{t}-\frac{1}{|\mathcal{G}|} \sum_{j \in \tilde{\mathcal{G}}} x_{j}^{t} \pi_{j t}^{s} \leq P_{i}^{\max }, \forall i \in \overline{\mathcal{G}} \\ 1 & \text { Otherwise }\end{cases}
$$

for the probabilistic constraints (28) and (29). Therefore, we rewrite (26), (27), (30), (31), (28) and (29) as the following constraints:

$$
\begin{array}{ll}
-\sum_{i \in \mathcal{D}} \hat{B}_{l i}^{d} d_{i}^{t}+\sum_{i \in \overline{\mathcal{G}}} \hat{B}_{l i}^{\bar{g}}\left(\bar{p}_{i}^{t}-\frac{1}{|\overline{\mathcal{G}}|} \sum_{j \in \tilde{\mathcal{G}}} x_{j}^{t} \pi_{j t}^{s}\right)+\sum_{j \in \tilde{\mathcal{G}}} \hat{B}_{l j}^{\tilde{g}} x_{j}^{t} \pi_{j t}^{s}-M \bar{z}_{s}^{t} \leq \bar{f}_{l} & \forall t, l \in \mathcal{L} \\
\sum_{i \in \mathcal{D}} \hat{B}_{l i}^{d} d_{i}^{t}-\sum_{i \in \overline{\mathcal{G}}} \hat{B}_{l i}^{\bar{g}}\left(\bar{p}_{i}^{t}-\frac{1}{|\overline{\mathcal{G}}|} \sum_{j \in \tilde{\mathcal{G}}} x_{j}^{t} \pi_{j t}^{s}\right)-\sum_{j \in \tilde{\mathcal{G}}} \hat{B}_{l j}^{\tilde{g}} x_{j}^{t} \pi_{j t}^{s}-M \bar{z}_{s}^{t} \leq \bar{f}_{l} & \forall t, l \in \mathcal{L} \\
\bar{r}_{i}^{t} \tau-\frac{1}{|\overline{\mathcal{G}}|} \sum_{j \in \tilde{\mathcal{G}}} x_{j}^{t} \pi_{j t}^{s}+\frac{1}{|\overline{\mathcal{G}}|} \sum_{j \in \tilde{\mathcal{G}}} x_{j}^{t-1} \pi_{j t-1}^{s}-M \tilde{z}_{s}^{t} \leq r_{i}^{\max } & \forall t, i \in \overline{\mathcal{G}} \\
-\bar{r}_{i}^{t} \tau+\frac{1}{|\overline{\mathcal{G}}|} \sum_{j \in \tilde{\mathcal{G}}} x_{j}^{t} \pi_{j t}^{s}-\frac{1}{|\overline{\mathcal{G}}|} \sum_{j \in \tilde{\mathcal{G}}} x_{j}^{t-1} \pi_{j t-1}^{s}-M \tilde{z}_{s}^{t} \leq-r_{i}^{m i n} & \forall t, i \in \overline{\mathcal{G}} \\
\bar{p}_{i}^{t}-\frac{1}{|\overline{\mathcal{G}}|} \sum_{j \in \tilde{\mathcal{G}}} x_{j}^{t} \pi_{j t}^{s}-M \hat{z}_{s}^{t} \leq P_{i}^{\max } & \forall t, i \in \overline{\mathcal{G}} \\
-\bar{p}_{i}^{t}+\frac{1}{|\overline{\mathcal{G}}|} \sum_{j \in \tilde{\mathcal{G}}} x_{j}^{t} \pi_{j t}^{s}-M \hat{z}_{s}^{t} \leq-P_{i}^{m i n} & \forall t, i \in \overline{\mathcal{G}} \\
\sum_{s=1}^{m} \bar{z}_{s}^{t} \leq m \gamma & \forall t \\
\sum_{s=1}^{m} \tilde{z}_{s}^{t} \leq m \gamma & \forall t
\end{array}
$$




$$
\sum_{s=1}^{m} \hat{z}_{s}^{t} \leq m \gamma
$$

Finally, we can write the deterministic version of the stochastic ramping optimization model as follows:

$$
\min \sum_{i \in \mathcal{G}} \sum_{t=1}^{T} \sum_{s=1}^{m} C_{p}\left(\bar{p}_{i}^{t}-\frac{1}{|\overline{\mathcal{G}}|} \sum_{j \in \tilde{\mathcal{G}}} x_{j}^{t} \pi_{j t}^{s}\right)+C_{r}\left(\bar{r}_{i}^{t} \tau-\frac{1}{|\overline{\mathcal{G}}|} \sum_{j \in \tilde{\mathcal{G}}} x_{j}^{t} \pi_{j t}^{s}-x_{j}^{t-1} \pi_{j t-1}^{s}\right)
$$

s.t.

$$
\begin{array}{ll}
\sum_{i \in \mathcal{D}} d_{i}^{t}-\sum_{j \in \tilde{\mathcal{G}}} x_{j}^{t} \mu_{j}^{t}-\sum_{i \in \overline{\mathcal{G}}} \bar{p}_{i}^{t}=0 & \forall t \\
-\sum_{i \in \mathcal{D}} \hat{B}_{l i}^{d} d_{i}^{t}+\sum_{i \in \overline{\mathcal{G}}} \hat{B}_{l i}^{\bar{g}}\left(\bar{p}_{i}^{t}-\frac{1}{|\overline{\mathcal{G}}|} \sum_{j \in \tilde{\mathcal{G}}} x_{j}^{t} \pi_{j t}^{s}\right)+\sum_{j \in \tilde{\mathcal{G}}} \hat{B}_{l j}^{\tilde{g}} x_{j}^{t} \pi_{j t}^{s}-M \bar{z}_{s}^{t} \leq \bar{f}_{l} & \forall t, l \in \mathcal{L} \\
\sum_{i \in \mathcal{D}} \hat{B}_{l i}^{d} d_{i}^{t}-\sum_{i \in \overline{\mathcal{G}}} \hat{B}_{l i}^{\bar{g}}\left(\bar{p}_{i}^{t}-\frac{1}{|\overline{\mathcal{G}}|} \sum_{j \in \tilde{\mathcal{G}}} x_{j}^{t} \pi_{j t}^{s}\right)-\sum_{j \in \tilde{\mathcal{G}}} \hat{B}_{l j}^{\tilde{g}} x_{j}^{t} \pi_{j t}^{s}-M \bar{z}_{s}^{t} \leq \bar{f}_{l} & \forall t, l \in \mathcal{L} \\
\bar{r}_{i}^{t} \tau-\frac{1}{|\overline{\mathcal{G}}|} \sum_{j \in \tilde{\mathcal{G}}} x_{j}^{t} \pi_{j t}^{s}+\frac{1}{|\overline{\mathcal{G}}|} \sum_{j \in \tilde{\mathcal{G}}} x_{j}^{t-1} \pi_{j t-1}^{s}-M \tilde{z}_{s}^{t} \leq r_{i}^{m a x} & \forall t, i \in \overline{\mathcal{G}} \\
-\bar{r}_{i}^{t} \tau+\frac{1}{|\overline{\mathcal{G}}|} \sum_{j \in \tilde{\mathcal{G}}} x_{j}^{t} \pi_{j t}^{s}-\frac{1}{|\overline{\mathcal{G}}|} \sum_{j \in \tilde{\mathcal{G}}} x_{j}^{t-1} \pi_{j t-1}^{s}-M \tilde{z}_{s}^{t} \leq-r_{i}^{m i n} & \forall t, i \in \overline{\mathcal{G}} \\
\bar{p}_{i}^{t}-\frac{1}{|\overline{\mathcal{G}}|} \sum_{j \in \tilde{\mathcal{G}}} x_{j}^{t} \pi_{j t}^{s}-M \hat{z}_{s}^{t} \leq P_{i}^{m a x} & \forall t, i \in \overline{\mathcal{G}} \\
-\bar{p}_{i}^{t}+\frac{1}{|\overline{\mathcal{G}}|} \sum_{j \in \tilde{\mathcal{G}}} x_{j}^{t} \pi_{j t}^{s}-M \hat{z}_{s}^{t} \leq-P_{i}^{m i n} & \forall t, i \in \overline{\mathcal{G}} \\
\sum_{s=1}^{m} \bar{z}_{s}^{t} \leq m \gamma & \\
\sum_{s=1}^{m} \tilde{z}_{s}^{t} \leq m \gamma & \forall t \\
\sum_{s=1}^{m} \hat{z}_{s}^{t} \leq m \gamma & \forall t \\
\bar{p}_{i}^{t}=\bar{p}_{i}^{t-1}+\bar{r}_{i}^{t} \tau & \forall t \\
0 \leq x_{j}^{t} \leq 1 & \forall t, i \in \overline{\mathcal{G}}
\end{array}
$$

